\title{
Extremal properties of the determinant of the Laplacian in the Bergman metric on the moduli space of genus two Riemann surfaces
}

\author{
C. Klein · A. Kokotov • D. Korotkin
}

Received: 10 December 2006 / Accepted: 28 October 2007 / Published online: 2 February 2008

(C) Springer-Verlag 2008

\begin{abstract}
We study extremal properties of the determinant of the Laplacian in the Bergman metric on the moduli space of compact genus two Riemann surfaces. By a combination of analytical and numerical methods we identify four non-degenerate critical points of this function and compute the signature of the Hessian at these points. The curve with the maximal number of automorphisms (the Burnside curve) turns out to be the point of the absolute maximum. Our results agree with the mass formula for virtual Euler characteristics of the moduli space. A similar analysis is performed for Bolza's strata of symmetric Riemann surfaces of genus two.
\end{abstract}

\section{Contents}

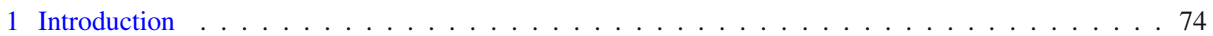

2 Summary of the genus one case . . . . . . . . . . . . . . . . . . . . 77

3 Moduli space of genus two Riemann surfaces . . . . . . . . . . . . . . . . . . . . . 78

$4 \mathcal{F}$ on the full moduli space of genus two curves . . . . . . . . . . . . . . . . . . . . . . 92

$5 \mathcal{F}$ on different strata of $\mathcal{M} \ldots \ldots \ldots \ldots$

$6 \mathcal{F}$ and Euler characteristics of moduli spaces . . . . . . . . . . . . . . . . . . . . . . 101

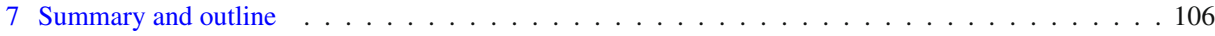

C. Klein

Max-Planck Institute for Mathematics in the Sciences, Inselstraße 22,

04103 Leipzig, Germany

Present Address:

C. Klein

Institut de Mathématiques de Bourgogne, Université de Bourgogne,

9 Avenue de A. Savary, BP 47970, 21078 Dijon Cedex, France

\section{A. Kokotov · D. Korotkin $(\varangle)$}

Department of Mathematics and Statistics, Concordia University, 7141 Sherbrooke West,

Montreal H4B 1R6, QC, Canada

e-mail: korotkin@crm.umontreal.ca 


\section{Introduction}

The study of extremal properties of various functionals related to Riemann surfaces attracted the attention of many researchers during the last 25 years (see [29] and other papers of the same volume [28] for an introduction to the subject). The functionals studied so far are related to both purely geometrical aspects of a Riemann surface as the function syst (the length of the shortest closed geodesic, see [31] and references therein), and to spectral aspects as the minimal eigenvalue of the corresponding Laplace operator [14], or the (appropriately regularized) determinant of the Laplacian [23]. The spectral characteristics are determined not only by the conformal structure on a Riemann surface, but also by the choice of a metric within a given conformal class. As was shown in [23] (for a short proof see [19]), the determinant of the Laplacian det $\Delta$ within a given conformal class of compact Riemann surfaces of fixed genus $g \geq 0$ and fixed volume takes its maximum for the metric of constant curvature; therefore this determinant defines a natural functional on the moduli space of Riemann surfaces.

So far the study of extremal properties of $\operatorname{det} \Delta$ in metrics of constant curvature did not go beyond the genus one case, where it is possible to prove that the tori with periods equal to $i$ and $e^{\pi i / 3}$ are critical points of $\operatorname{det} \Delta$ on the moduli space: $e^{\pi i / 3}$ is the maximum, and $i$ is the saddle point. The proof of the vanishing of the gradient of $\operatorname{det} \Delta$ at these two points is based on the existence of subgroups of the modular group leaving these points invariant [23] (i.e., the corresponding elliptic surfaces have non-trivial automorphisms groups); thus the gradient of any modular invariant functional, not only det $\Delta$, vanishes at these two points.

In genus two and higher the analysis of extremal properties of any functional becomes decisively more complicated. Nonetheless some explicit results are known in genera two and three (it was recently proved in [14] that the minimal eigenvalue of the Laplacian in genus two is maximal on the Burnside curve equipped with a singular metric with cone singularities); the same holds for the function syst [31] (the Jacobian of the Burnside curve also defines a lattice in $\mathbb{C}^{2}$ corresponding to the densest sphere packing [27]). In [31] syst was used as a topological Morse function to define a cell decomposition of the moduli spaces; in the simplest cases (in genera 0,1,2 with a small number of punctures) the analysis of the critical points of syst allowed to reproduce known values of virtual Euler characteristics of the moduli spaces [11] via the mass formula.

Unfortunately any reasonably complete treatment of extremal properties of the determinant of the Laplacian in the Poincaré metric in genus two seems to be out of reach at the moment due to the absence of efficient numerical algorithms for the computation of this determinant (perhaps such an algorithm can be developed by extending results of [24] to the full moduli space).

The goal of this paper is to study extremal properties of another smooth functional on the moduli space of genus two Riemann surfaces with good boundary behavior-the function

$$
\mathcal{F}=(\operatorname{det} \Im \mathbf{B})^{5 / 2} \prod_{s=1}^{10}\left|\Theta\left[\beta_{s}\right](\mathbf{B})\right|
$$

The objects entering the expression (1.1) are defined as follows. Consider a canonical basis of cycles $\left(a_{j}, b_{j}\right), \quad j=1,2$ on a Riemann surface $\mathcal{L}$ of genus 2 with the intersection indices $a_{i} \circ a_{j}=b_{i} \circ b_{j}=0 ; a_{i} \circ b_{j}=\delta_{i j}$. The dual basis of holomorphic 1-forms $v_{k}$ on $\mathcal{L}$ is normalized by $\oint_{a_{j}} v_{k}=\delta_{j k}$. The matrix of $b$-periods of $\mathcal{L}$, which contains the complete information about the complex structure of $\mathcal{L}$, is defined by $\mathbf{B}_{j k}=\oint_{b_{j}} v_{k}$. 
An even theta-characteristic $\beta_{s}$ is a pair of vectors $\mathbf{p}_{s}, \mathbf{q}_{s}$ from $\mathbb{R}^{2}$ (in genus 2) with half-integer entries such that the integer number $4\left\langle\mathbf{p}_{s}, \mathbf{q}_{s}\right\rangle$ is even. The theta-constant corresponding to the characteristic $\beta_{s}$ is defined as follows:

$$
\Theta\left[\beta_{s}\right](\mathbf{B})=\sum_{\mathbf{m} \in \mathbf{Z}^{2}} \exp \left\{\pi i\left\langle\mathbf{B}\left(\mathbf{m}+\mathbf{p}_{s}\right),\left(\mathbf{m}+\mathbf{p}_{s}\right)\right\rangle+2 \pi i\left\langle\mathbf{q}_{s},\left(\mathbf{m}+\mathbf{p}_{s}\right)\right\rangle\right\}
$$

(in this paper we denote by $\Theta$ the genus two theta function; the genus one theta functions are denoted by $\vartheta$ ).

The expression (1.1) where the product is taken over all ten even theta characteristics $\beta_{s}$, does not depend on the choice of canonical basis of cycles on $\mathcal{L}$.

The function $\mathcal{F}$ is related to the determinant of the Laplace operator as follows [37]:

$$
\operatorname{det} \Delta_{B}=C \mathcal{F}^{1 / 3},
$$

where $C$ is a moduli-independent constant; $\Delta_{B}$ is the Laplacian in the Bergman metric- the metric of volume 1 given by

$$
g_{B}:=\frac{1}{2} \sum_{j, k=1}^{2}(\Im \mathbf{B})_{j k}^{-1} v_{j} \bar{v}_{k}
$$

The Bergman metric (also called sometimes "canonical metric" [15, p. 461]) is induced on $\mathcal{L}$ by the standard flat invariant Kähler metric on the Jacobian when $\mathcal{L}$ is canonically embedded into its Jacobian via the Abel map. The Bergman metric is also related to the Bergman reproducing kernel $B(P, Q):=\sum_{j, k=1}^{2}(\Im B)_{j k}^{-1} v_{j}(P) \bar{v}_{k}(Q)$ for holomorphic differentials; being taken on the diagonal the Bergman kernel gives the Bergman metric (1.3). ${ }^{1}$

Since in genus two the theta divisor can be biholomorphically mapped to the Riemann surface itself, $\mathcal{F}^{-1 / 3}$ turns out to coincide also with the analytic torsion of the theta divisor equipped with the metric induced by the same Kähler metric on the Jacobian [36].

The function $\mathcal{F}$ is known to appear also in several other contexts. Being considered as a function on the upper Siegel half-space $\mathcal{H}$, the function $\mathcal{F}$ coincides with the Petersson norm $\left\|\Delta_{2}\right\|=(\operatorname{det} \Im \mathbf{B})^{5 / 2}\left|\Delta_{2}\right|$ of the Siegel cusp form $\Delta_{2}:=\prod_{s=1}^{10} \Theta\left[\beta_{s}\right]$. The function $\mathcal{F}$ essentially coincides also with the genus two Mumford measure [16]. Finally, as it was recently noticed by Yoshikawa [36,37], the function $\mathcal{F}$ coincides with the analytic torsion invariant of the corresponding Kummer surface.

On the boundary of the moduli space $\mathcal{M}$ of genus two Riemann surfaces $\mathcal{F}$ vanishes; therefore $-\log \mathcal{F}$ is the proper function on $\mathcal{M}$; moreover, all of its critical points are nondegenerate (which is equivalent to the non-degeneracy of critical points of $\mathcal{F}$ itself), which together with the boundary behavior $-\log \mathcal{F} \rightarrow+\infty$ would allow the study of topological properties of $\mathcal{M}$.

In this paper we study critical points of $\mathcal{F}$ on $\mathcal{M}$ by using a combination of analytical and numerical tools. The function $\mathcal{F}$ can be also considered as a function on the Siegel upper half-space $\mathcal{H}$ invariant under the action of the group $\operatorname{Sp}(4, \mathbf{Z})$.

In particular, we prove the following theorem:

Theorem 1 The function $\mathcal{F}$, considered as a function on $\mathcal{H}$, has critical points corresponding to matrices of b-periods of three curves with large automorphizm groups: the Burnside curve

\footnotetext{
1 There exists a terminological inconsistency in the literature: from analogy to the theory of several complex variables [3, p. 122] it is natural to use the name "Bergman metric" for the metric given by the curvature of the metric (1.3). On the other hand, according to terminology which we follow here (1.3) itself is also called Bergman metric [35, p. 490] and [22, p. 317].
} 
$y^{2}=x\left(x^{4}-1\right)$, the curve $y^{2}=x^{6}-1$ and the curve $y^{2}=x^{5}-1$. In addition, there are critical points corresponding to the matrices of $b$-periods of some curve lying within the family $y^{2}=\left(z^{3}-1\right)\left(z^{3}-r^{3}\right)$.

Further numerical analysis of $\mathcal{F}$ in Gottschling's fundamental domain $\mathcal{H} / \mathrm{Sp}(4, \mathbf{Z})$ shows that all of these critical points are non-degenerate, that the Burnside curve gives the absolute maximum of $\mathcal{F}$, and that the signature of the Hessian at these three points is equal to $(0,6)$, $(3,3)$ and $(2,4)$, respectively. In addition the numerical analysis shows the existence of the fourth critical point-a curve from the family $y^{2}=\left(z^{3}-1\right)\left(z^{3}-r^{3}\right)$ with

$$
r=0.22373907612077 \ldots
$$

This point is also non-degenerate, and the signature of the Hessian there is $(1,5)$. The numerical analysis also shows the absence of other critical points.

These numerical results support the following conjecture:

Conjecture 1 Apart from three curves with large automorphizm groups, there exists one and only one other critical point of $\mathcal{F}$ on $\mathcal{M}$, which belongs to the family $y^{2}=\left(z^{3}-1\right)\left(z^{3}-r^{3}\right)$.

Though the presence of the fourth critical point appears somewhat unexpected at first, its existence is predicted by the mass formula [31] for orbifold Euler characteristics of $\mathcal{M}$. The mass formula states that the Euler characteristic is equal to the sum over all critical Riemann surfaces $\mathcal{L}_{i}$ of $(-1)^{c_{i}} /\left\{\# A u t\left(\mathcal{L}_{i}\right)\right\}$ where $c_{i}$ is the index of the critical point; \#Aut $\left(\mathcal{L}_{i}\right)$ is the order of the group of automorphisms of $\mathcal{L}_{i}$. One can easily check that the mass formula immediately implies the existence of the fourth critical curve.

Actually, in Theorem 6 we show that any $\operatorname{Sp}(4, \mathbf{Z})$-invariant function on $\mathcal{H}$ which is $C^{1}$ in vicinities of points corresponding to matrices of $b$-periods of the three curves with large automorphizm groups listed in Theorem 1 has vanishing gradient at these points. Besides $\mathcal{F}$, other examples of such functions are given by determinants of Laplacians in Poincaré and Arakelov metrics, as well as by values of the corresponding operator zeta-functions at any point.

We perform a similar analysis on three smaller moduli spaces of genus two curves with fixed groups of automorphisms: the space of (complex) dimension two of curves with a $\mathbf{Z}_{2} \times \mathbf{Z}_{2}$ symmetry group, and one-dimensional spaces of curves with $\mathbf{Z}_{2} \times D_{2}$ and $\mathbf{Z}_{2} \times D_{3}$ symmetry groups; it turns out that $\mathcal{F}$ does not have any new critical points on these subspaces. In particular, we show that the moduli space of $\mathbf{Z}_{2} \times D_{2}$ curves in coordinates given by the one remaining parameter in the matrix of $b$-periods can be identified with the factor of the upper half-plane by the modular group $\Gamma_{0}(2)+$; similarly, the moduli space of $\mathbf{Z}_{2} \times D_{3}$ curves can be identified with the factor of the upper half-plane by the modular group $\Gamma_{0}(3)+$. We discuss the meaning of the mass formula for these subspaces of symmetric curves.

The paper is organized as follows: In Sect. 2 we recall what is known about extremal properties of det $\Delta$ on the moduli space of genus one Riemann surfaces. In Sect. 3 we summarize necessary facts on the description of the full moduli space, as well as of its symmetric strata, in terms of matrices of $b$-periods. Although most of the facts presented here are well-known, we did not find some of them (about the link between $D_{2}$ and $D_{3}$ moduli spaces with subgroups $\Gamma_{0}(2)+$ and $\Gamma_{0}(3)+$ of the modular group) in the existing literature. In Sect. 4 we prove that the three curves with large automorphism groups are stationary points of $\mathcal{F}$, as well as of any other smooth function on $\mathcal{H}$ invariant under the action of the $\operatorname{Sp}(4, \mathbf{Z})$ group. Numerical analysis shows the existence of an additional critical point on $\mathcal{M}$ and the non-degeneracy of all four critical points. The Burnside curve turns out to be the global maximum of $\mathcal{F}$ on $\mathcal{M}$; we compute the signature of the Hessian for the other critical points. In Sect. 5 a similar 
analysis is performed for the three strata of $\mathcal{M}$ with given degree of symmetry. In Sect. 6 we outline the relationship of our results to the computation of virtual Euler characteristics of $\mathcal{M}$ and of its symmetric subspaces.

In the sequel we shall use the following notation for the root of unity: $\epsilon_{k}=e^{2 \pi i / k}$; the modular group $S L(2, \mathbf{Z})$ will be denoted by $\Gamma$.

\section{Summary of the genus one case}

In genus one the Bergman metric (1.3) coincides with the metric of constant curvature-the flat metric of volume 1 given by $d z d \bar{z} / \Im \sigma$ on the torus with periods 1 and $\sigma$. The determinant of the Laplacian (acting on functions, i.e., sections of the trivial line bundle) in this metric is, up to a multiplicative constant, given by the expression [26]:

$$
f(\sigma)=C(\Im \sigma)^{1 / 2}|\eta(\sigma)|^{2},
$$

where $\eta=\left[\vartheta_{1}^{\prime}\right]^{1 / 3}$ is the Dedekind eta-function; $C$ is a constant independent of the moduli. Due to the Jacobi formula $\vartheta_{1}^{\prime}=i \vartheta_{2} \vartheta_{3} \vartheta_{4}\left(\vartheta_{j}, j=2,3,4\right.$ are the standard theta-constants) the expression (2.1) is a straightforward analog of the genus two expression (1.1).

The function $f(2.1)$ is modular invariant, real and positive. Moreover it vanishes on the boundary of the moduli space, when the torus degenerates and when $\sigma$ tends to $+i \infty$ (or any other point related to $+i \infty$ by a modular transformation). The function $f$ has the following obvious symmetry with respect to reflections at the imaginary axis:

$$
f(-\bar{\sigma})=f(\sigma)
$$

The extremal properties of the function $f(2.1)$ are well-known (see [23,27]):

Theorem 2 The points $\sigma=i$ and $\sigma=e^{\pi i / 3}$ (as well as all points obtained from these two points by modular transformations) are stationary points of the function (2.1); the point $e^{\pi i / 3}$ is the point of the absolute maximum of $f$.

Both points $i$ and $e^{\pi i / 3}$ are orbifold points (with cone angle $\pi$ and $2 \pi / 3$, respectively) of the moduli space which can be obtained by an appropriate identification of the boundary of the fundamental domain $\Omega$ of the group $\Gamma$ : there are non-trivial subgroups of the modular group leaving these two points invariant. Introduce the standard generators of $\Gamma$ :

$$
t=\left(\begin{array}{ll}
1 & 1 \\
0 & 1
\end{array}\right), \quad s=\left(\begin{array}{cc}
0 & -1 \\
1 & 0
\end{array}\right) .
$$

The stationary subgroup of order 2 leaving the point $\sigma=i$ invariant is generated by the element $s$. The stationary subgroup of order 3 leaving the point $\sigma=e^{\pi i / 3}$ invariant is generated by the product $s t$.

The proof of the stationarity [23] of $f(\sigma)$ at these two points works equally well for any smooth function on the upper half-plane invariant under the modular group; below we prove a similar statement in the genus two case. It was proved analytically in [23] that the point $e^{\pi i / 3}$ is the local maximum of $f(\sigma)$; it can be shown numerically that actually the point $e^{\pi i / 3}$ is the absolute maximum, and $i$ is the saddle point of $f$ (see Fig. 1 for the plot of $f$ in the fundamental domain $\Omega$ of the modular group).

It is instructive to plot $f$ also as a function of the $J$-invariant:

$$
J(\sigma):=\frac{\left(\vartheta_{2}^{8}+\vartheta_{3}^{8}+\vartheta_{4}^{8}\right)^{3}}{54 \vartheta_{2}^{8} \vartheta_{3}^{8} \vartheta_{4}^{8}},
$$




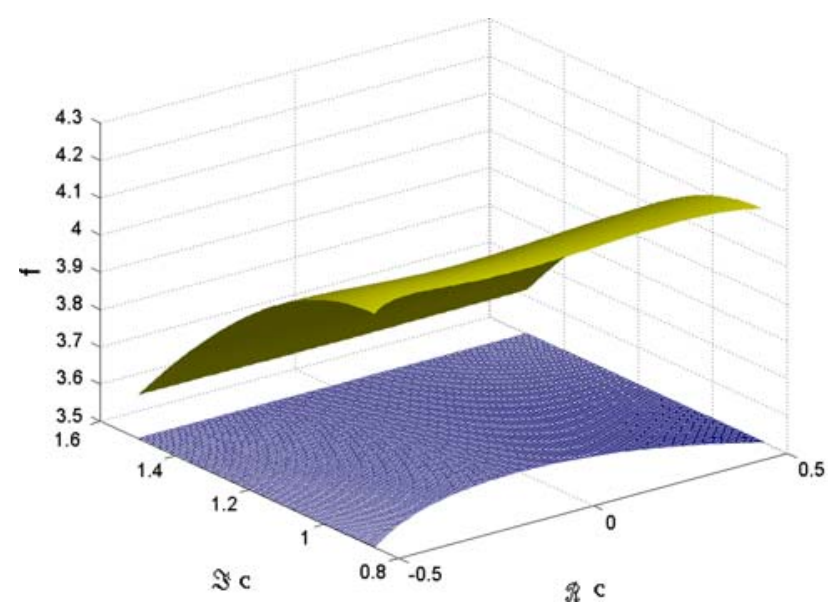

Fig. 1 Plot of $f(\sigma)$ in the fundamental domain $\Omega$

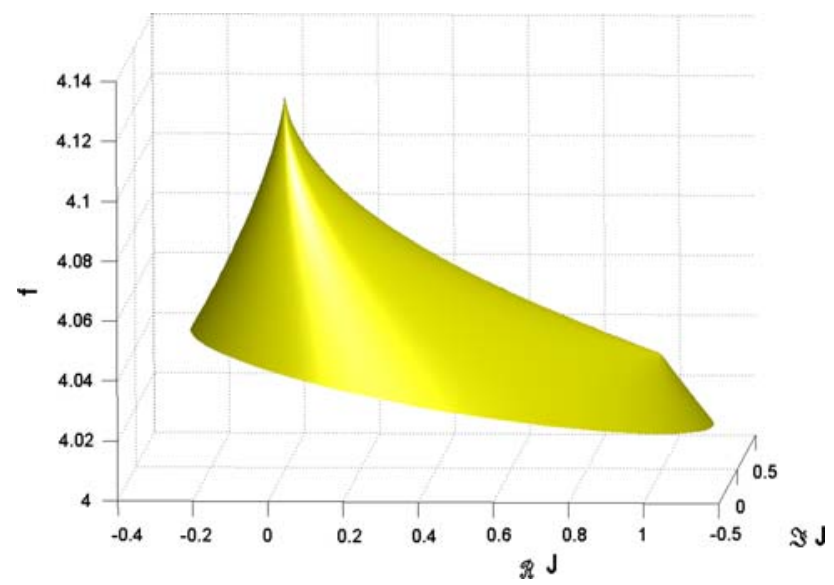

Fig. 2 Plot of $f$ as a function of the $J$-invariant

which maps the fundamental domain $\Omega$ onto the whole complex plane. The maximum of $f$ in Fig. 2 is achieved at $J\left(e^{\pi i / 3}\right)=0$ (the highest peak); the spike at $J(i)=1$ is the saddle point. The spikes of the function $f(J)$ at these points appear since the change of coordinates $\sigma \rightarrow J$ is degenerate there.

\section{Moduli space of genus two Riemann surfaces}

\subsection{The Siegel fundamental domain: Gottschling's description}

The moduli space of Riemann surfaces $\mathcal{M}$ is covered (with branching) by the Torelli space $\mathcal{T}$ which is the space of marked Riemann surfaces, i.e., the space of pairs (the Riemann surface $\mathcal{L}$ and the canonical basis of cycles on $\mathcal{L}$ ). The Torelli space is still not simply connected; 
its fundamental group is called the Torelli group; the universal covering of the Torelli space is the Teichmüller space. It is important for us that the covering of the Torelli space by the Teichmüller space is unramified, i.e., that any analytic function on the Torelli space remains analytic whilst being lifted to the Teichmüller space.

Consider the upper Siegel half-space $\mathcal{H}=\left\{z \in M(2, \mathbb{C}): z=z^{t} ; \mathfrak{s} z \geq 0\right\}$. Assigning to any marked Riemann surface its matrix of $b$-periods computed in a given basis, we get the map from the Torelli space to $\mathcal{H}$; the change of a canonical basis of cycles on a given Riemann surface corresponds to an $\operatorname{Sp}(4, \mathbf{Z})$ transformation of $\mathcal{H}$. The image of this map does not coincide with the whole space $\mathcal{H}$ since the matrix of $b$-periods of a non-degenerate Riemann surface of genus two can never be diagonal or equivalent to diagonal up to a modular transformation. Denote by $\mathcal{D} \subset \mathcal{H}$ the set of matrices which are either diagonal or can be transformed to diagonal form by a modular transformation. In genus two, when the independent entries of the matrix of $b$-periods can be used as local coordinates on the moduli space, the space $\mathcal{H} \backslash \mathcal{D}$ can be identified with the Torelli space. The factor of $\mathcal{H} \backslash \mathcal{D}$ by the action of $\operatorname{Sp}(4, \mathbf{Z})$ can be identified with the moduli space $\mathcal{M}$ of genus two Riemann surfaces.

We can also first factorize $\mathcal{H}$ by the action of $\operatorname{Sp}(4, \mathbf{Z})$. This gives the Siegel-Gottschling fundamental domain $\mathcal{G}:=\mathcal{H} / \operatorname{Sp}(4, \mathbf{Z})$; taking out from $\mathcal{G}$ points lying in $\mathcal{D}$, we get the space $\mathcal{G} \backslash \mathcal{D}$ which also coincides with the moduli space $\mathcal{M}$.

The fundamental domain $\mathcal{G}$ can be described by 25 inequalities on the matrix entries of $\mathbf{B}$ [10]. To describe these conditions we introduce real and imaginary parts of the independent components of $\mathbf{B}: \mathbf{B}_{11}=x_{1}+i y_{1}, \mathbf{B}_{12}=x_{2}+i y_{2}$ and $\mathbf{B}_{22}=x_{3}+i y_{3}$.

Then $\mathcal{G}$ is defined by the following set of inequalities:

- Conditions restricting the range of $\left\{x_{i}\right\}$ and $\left\{y_{i}\right\}$ :

$$
\left|x_{i}\right| \leq \frac{1}{2}, \quad i=1,2,3, \quad y_{i} \geq \frac{1}{2} \sqrt{3}, \quad i=1,3, \quad y_{2} \geq 0 .
$$

- The Minkowski ordering condition:

$$
y_{1} \geq 2 y_{2}, \quad y_{3} \geq y_{1},
$$

- The following set of 19 inequalities:

$$
\left|\mathbf{B}_{11}\right| \geq 1, \quad\left|\mathbf{B}_{22}\right| \geq 1, \quad\left|\mathbf{B}_{11}+\mathbf{B}_{22}-2 \mathbf{B}_{12}+\mathbf{e}\right| \geq 1
$$

and

$$
|\operatorname{det}(\mathbf{B}+S)| \geq 1
$$

where $S$ are the matrices

$$
\begin{gathered}
\left(\begin{array}{ll}
0 & 0 \\
0 & 0
\end{array}\right),\left(\begin{array}{ll}
\mathbf{e} & 0 \\
0 & 0
\end{array}\right),\left(\begin{array}{ll}
0 & 0 \\
0 & \mathbf{e}
\end{array}\right),\left(\begin{array}{ll}
\mathbf{e} & 0 \\
0 & \mathbf{e}
\end{array}\right), \\
\left(\begin{array}{cc}
\mathbf{e} & 0 \\
0 & -\mathbf{e}
\end{array}\right),\left(\begin{array}{ll}
0 & \mathbf{e} \\
\mathbf{e} & 0
\end{array}\right),\left(\begin{array}{ll}
\mathbf{e} & \mathbf{e} \\
\mathbf{e} & 0
\end{array}\right),\left(\begin{array}{ll}
0 & \mathbf{e} \\
\mathbf{e} & \mathbf{e}
\end{array}\right)
\end{gathered}
$$

and $\mathbf{e}= \pm 1$

\subsection{Curves with non-trivial automorphisms: Bolza's classification}

Any genus two Riemann surface is biholomorphically equivalent to an algebraic curve defined by an equation: $y^{2}=f(z)$ where $f(z)$ is a polynomial of degree 5 or 6 . The hyperelliptic 
involution on this curve maps any point $(y, z)$ to $(-y, z)$; this involution generates the hyperelliptic symmetry group $\mathbf{Z}_{2}$. A generic genus two curve does not have other automorphisms. If a curve has a larger automorphism group $A u t$, the hyperelliptic $\mathbf{Z}_{2}$ subgroup always turns out to be a normal subgroup of Aut. All symmetric curves can be stratified according to the type of the reduced automorphism group $A u t / \mathbf{Z}_{2}$ which can be one of the following six types [4]: $\mathbf{Z}_{2}, D_{2}, D_{3}, S_{4}, D_{6}$ or $\mathbf{Z}_{5}$ (where $D_{i}$ are dihedral groups).

\subsubsection{Group $\mathbf{Z}_{2}$}

Any Riemann surface $\mathcal{L}$ from this family can be represented by the equation

$$
y^{2}=\left(z^{2}-1\right)\left(z^{2}-r_{1}^{2}\right)\left(z^{2}-r_{2}^{2}\right)
$$

where $r_{1}, r_{2} \in \mathbb{C}$. In addition to the hyperelliptic involution there is an involution $\mu$ acting as $\mu: z \rightarrow-z$ on the curve (3.6). The basic cycles on $\mathcal{L}$ chosen as shown in Fig. 3 transform as follows under the action of the involution $\mu$ :

$$
a_{1}^{\mu}=a_{2} \quad b_{1}^{\mu}=b_{2},
$$

or equivalently,

$$
\left(\begin{array}{l}
b_{1}^{\mu} \\
b_{2}^{\mu} \\
a_{1}^{\mu} \\
a_{2}^{\mu}
\end{array}\right)=T^{\mu}\left(\begin{array}{l}
b_{1} \\
b_{2} \\
a_{1} \\
a_{2}
\end{array}\right)
$$

where the $\operatorname{Sp}(4, \mathbf{Z})$ matrix $T^{\mu}$ is given by

$$
T^{\mu}=\left(\begin{array}{llll}
0 & 1 & 0 & 0 \\
1 & 0 & 0 & 0 \\
0 & 0 & 0 & 1 \\
0 & 0 & 1 & 0
\end{array}\right)
$$

Writing the matrix $T^{\mu}$ in block form:

$$
T^{\mu}=\left(\begin{array}{ll}
A^{\mu} & B^{\mu} \\
C^{\mu} & D^{\mu}
\end{array}\right)
$$

where $B^{\mu}=C^{\mu}=0 ; A^{\mu}=D^{\mu}=\left(\begin{array}{ll}0 & 1 \\ 1 & 0\end{array}\right)$, and taking into account that, on one hand, the matrix of $b$-periods transforms under the action of any symplectic transformation as follows:

$$
\mathbf{B} \rightarrow \mathbf{B}^{\mu}:=\left(A^{\mu} \mathbf{B}+B^{\mu}\right)\left(C^{\mu} \mathbf{B}+D^{\mu}\right)^{-1},
$$

and that, on the other hand, the matrix of $b$-periods must remain invariant under the action of the biholomorphic transformation mapping the canonical basis of cycles to the new one, we conclude that $\mathbf{B}=\mathbf{B}^{\mu}$. This is equivalent to

$$
\mathbf{B}=\left(\begin{array}{ll}
0 & 1 \\
1 & 0
\end{array}\right) \mathbf{B}\left(\begin{array}{ll}
0 & 1 \\
1 & 0
\end{array}\right) ;
$$

thus the matrix $\mathbf{B}$ can be parametrized as follows:

$$
\mathbf{B}=\frac{1}{2}\left(\begin{array}{ll}
\mathbf{x}+\mathbf{y} & \mathbf{x}-\mathbf{y} \\
\mathbf{x}-\mathbf{y} & \mathbf{x}+\mathbf{y}
\end{array}\right)
$$




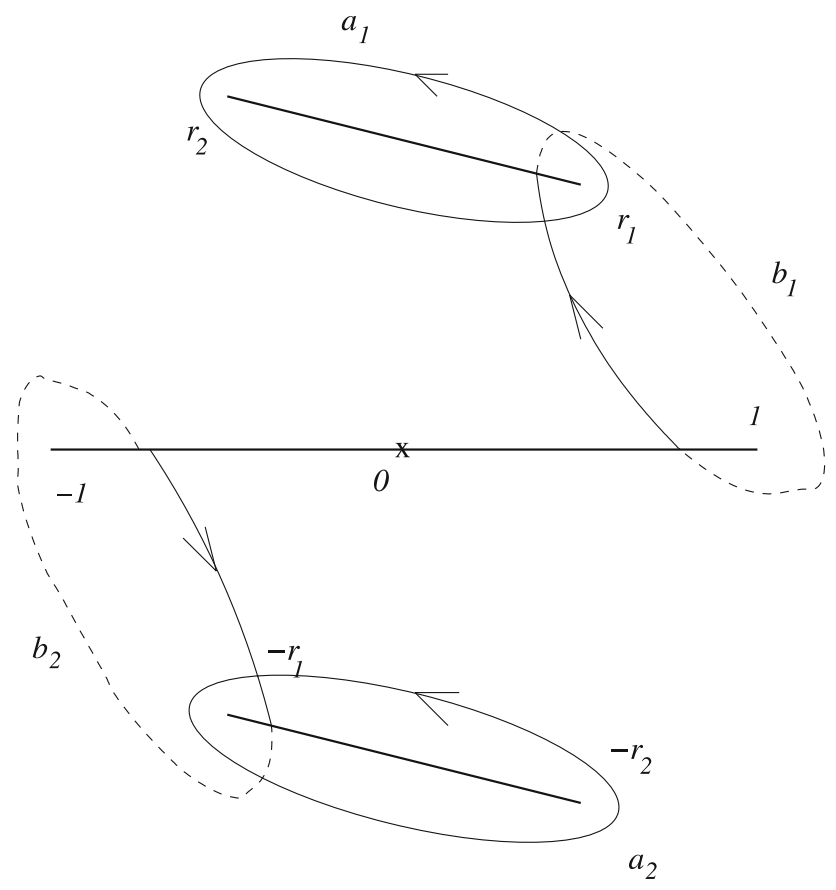

Fig. 3 Canonical basis of cycles on a curve with $\mathbf{Z}_{2} \times \mathbf{Z}_{2}$ symmetry

If we factorize $\mathcal{L}$ with respect to the involution $\mu$, we get an elliptic curve $\mathcal{L}_{0}$ with period $\mathbf{y}$; $\mathbf{x}$ is the period of the elliptic Prym variety corresponding to the (ramified) covering $\mathcal{L} \rightarrow \mathcal{L}_{0}$.

The description of the moduli space $\mathcal{M}\left(\mathbf{Z}_{2}\right)$ was given in [29] in terms of the variables $\tau_{1}=\mathbf{y}$ and $\tau_{2}=-1 / \mathbf{x}$. Below we describe this construction in terms of $\mathbf{x}$ and $\mathbf{y}$ themselves which makes it slightly more transparent. Denote by $\Gamma(2)$ the main congruence subgroup of $\Gamma$ consisting of matrices $\gamma$ such that $\gamma \equiv I(\bmod 2)(I$ is the unit matrix).

The natural idea of [29] is to reduce the action of the full group $\operatorname{Sp}(4, \mathbf{Z})$ on matrices of the form (3.12) to a correlated action of $\Gamma$ on $\mathbf{x}$ and $\mathbf{y}$.

Lemma 1 Assume that the basis of canonical cycles $\left(b_{1}, b_{2}, a_{1}, a_{2}\right)$ satisfying (3.7) is transformed by a matrix $T \in \mathrm{Sp}(4, \mathbf{Z})$ to another basis $\left(b_{1}^{\prime}, b_{2}^{\prime}, a_{1}^{\prime}, a_{2}^{\prime}\right)$ satisfying the same relation:

$$
a_{1}^{\prime \mu}=a_{2}^{\prime}, \quad b_{1}^{\prime \mu}=b_{2}^{\prime} .
$$

Then the action of the matrix $T$ on the matrix of b-periods (3.12) gives rise to an action of two elements $\gamma_{1}, \gamma_{2} \in \Gamma$, such that $\gamma_{1} \gamma_{2}^{-1} \in \Gamma(2)$, on $\mathbf{x}$ and $\mathbf{y}$, respectively. The matrix $T$ is expressed in terms of $\gamma_{1,2}$ as follows: if

$$
\gamma_{i}=\left(\begin{array}{cc}
k_{i} & l_{i} \\
m_{i} & n_{i}
\end{array}\right), \quad i=1,2
$$

then

$$
T=\frac{1}{2}\left(\begin{array}{cccc}
k_{1}+k_{2} & k_{1}-k_{2} & l_{1}+l_{2} & l_{1}-l_{2} \\
k_{1}-k_{2} & k_{1}+k_{2} & l_{1}-l_{2} & l_{1}+l_{2} \\
m_{1}+m_{2} & m_{1}-m_{2} & n_{1}+n_{2} & n_{1}-n_{2} \\
m_{1}-m_{2} & m_{1}+m_{2} & n_{1}-n_{2} & n_{1}+n_{2}
\end{array}\right)
$$


Proof The additional symmetries of the matrix $T \in \mathrm{Sp}(4, \mathbf{Z})$ (3.15) follow from the Assumptions (3.7), (3.13) about the behavior of the basic cycles under the action of the involution $\mu$. The equivalence of the action of the matrix $T$ on the matrix (3.12) to the action of the two elements $\gamma_{1,2}$ (3.14) separately on $\mathbf{x}$ and $\mathbf{y}$ follows by direct computation. Finally the condition that all matrix entries of $T$ (3.15) are integer is equivalent to the condition that all matrix entries of the matrix $\gamma_{1}-\gamma_{2}$ are even. A simple computation using the conditions $\operatorname{det} \gamma_{i}=1$ shows that this is equivalent to the condition $\gamma_{1} \gamma_{2}^{-1} \in \Gamma(2)$ (which is more natural from the point of view of the group structure); it is straightforward to check that this condition is invariant under the natural group operation in $\Gamma \times \Gamma$.

As was proved in [29], to get the full subgroup of $\operatorname{Sp}(4, \mathbf{Z})$ preserving the form (3.12) of the matrix of $b$-periods, one has to add to the subgroup (3.15) one more transformation, given by

$$
T=\left(\begin{array}{cccc}
1 & 0 & 0 & 0 \\
0 & -1 & 0 & 0 \\
0 & 0 & 1 & 0 \\
0 & 0 & 0 & -1
\end{array}\right)
$$

which maps the canonical basis $\left(b_{1}, b_{2}, a_{1}, a_{2}\right)$ to the basis $b_{1}^{\prime}=b_{1}, b_{2}^{\prime}=-b_{2}$, and $a_{1}^{\prime}=$ $a_{1}, a_{2}^{\prime}=-a_{2}$; obviously, this transformation destroys the symmetry (3.7) of the canonical basis of cycles under the involution $\mu$. The action of the transformation (3.16) on the matrix of $b$-periods (3.12) is very simple: it changes the sign of the off-diagonal terms, i.e., it corresponds to the interchange of $\mathbf{x}$ and $\mathbf{y}: \mathbf{x}^{\prime}=\mathbf{y}, \mathbf{y}^{\prime}=\mathbf{x}$. Let us denote by $S_{2}$ the twoelement permutation group generated by this transformation.

The theorem proved in [29] can now be reformulated as follows:

Theorem 3 The moduli space $\mathcal{M}\left(\mathbf{Z}_{2}\right)$ can be represented as the following factor:

$$
\mathcal{M}\left(\mathbf{Z}_{2}\right)=\mathcal{S}\left(\mathbf{Z}_{2}\right) / G
$$

where

$$
\mathcal{S}\left(\mathbf{Z}_{2}\right)=H \times H \backslash\{(\mathbf{x}, \mathbf{y}) \mid \mathbf{x}=\gamma \mathbf{y}, \mathbf{x}, \mathbf{y} \in H, \gamma \in \Gamma(2)\}
$$

( $H$ is the upper half-plane), and where the group $G$ is defined as follows:

$$
G=G_{0} S_{2}
$$

where $G_{0} S_{2}$ is the semi-direct product of two groups and $G_{0}$ is the following normal subgroup of $G$ :

$$
G_{0}=\left\{\left(\gamma_{1}, \gamma_{2}\right) \in \Gamma \times \Gamma \mid \gamma_{1} \gamma_{2}^{-1} \in \Gamma(2)\right\}
$$

The subspace $\{(\mathbf{x}, \mathbf{y}) \mid \mathbf{x}=\gamma \mathbf{y}, \mathbf{x}, \mathbf{y} \in H, \gamma \in \Gamma(2)\}$ is taken out of $H \times H$ since it consists of matrices which are $\operatorname{Sp}(4, \mathbf{Z})$ equivalent to diagonal ones. The appearance of the group $G$ is explained above; Lemma 3.15 shows how to construct the natural group homomorphism $f$ from $G$ to $\operatorname{Sp}(4, \mathbf{Z})$.

The space $\mathcal{S}\left(\mathbf{Z}_{2}\right)$ (3.18) is called in [29] the "special Torelli space", and the group $G$ (3.19) the "special Torelli group".

The non-trivial part of the proof of this theorem (for which we refer the reader to [29]) is to show that no $\operatorname{Sp}(4, \mathbf{Z})$ transformation from the complement of the image of the homomorphism $f$ preserves the matrix of $b$-periods (3.12). 
The structure of the fundamental domain $\mathcal{M}\left(\mathbf{Z}_{2}\right)$ (3.17) is rather non-trivial due to the necessity to take into account the subgroup $S_{2}$ interchanging $\mathbf{x}$ and $\mathbf{y}$. Therefore for our subsequent numerical analysis we introduce the "bigger" factor space $\Omega\left(\mathbf{Z}_{2}\right)=(H \times H) / G_{0}$, which can be easily described.

Lemma 2 The fundamental domain for the action of the group $\mathrm{G}_{0}$ on $\mathrm{H} \times \mathrm{H}$ can be chosen to be $\Omega \times \Omega(2)$, where $\Omega$ is the standard fundamental domain of the group $\Gamma$ (Fig. 4), and $\Omega(2)$ (Fig.5) is the fundamental domain of the subgroup $\Gamma(2)$, consisting of six copies of $\Omega$.

Proof is simple. To prove that any point $(\mathbf{x}, \mathbf{y}) \in H \times H$ can be mapped to the inside of $\Omega \times \Omega(2)$ by some transformation from $G_{0}$, we first identify a $\gamma_{1}$ such that $\gamma_{1} \mathbf{x} \in \Omega$. As the second step we find a transformation $\gamma \in \Gamma(2)$ such that $\gamma\left(\gamma_{1} \mathbf{y}\right) \in \Omega(2)$. Obviously the transformation mapping $(\mathbf{x}, \mathbf{y})$ to $\left(\gamma_{1} \mathbf{x}, \gamma \gamma_{1} \mathbf{y}\right)$ belongs to $G_{0}$ since $\gamma_{1}\left(\gamma \gamma_{1}\right)^{-1}=\gamma^{-1} \in$ $\Gamma(2)$.

Suppose now that some transformation $\left(\gamma_{1}, \gamma_{2}\right) \in G_{0}$ maps some point $(\mathbf{x}, \mathbf{y}) \in \Omega \times \Omega(2)$ to another point $\left(\mathbf{x}^{\prime}, \mathbf{y}^{\prime}\right) \in \Omega \times \Omega(2)$. Since $\gamma_{1} \in \Gamma$, it must be the identity element (since $\Omega$ is a fundamental domain for $\Gamma$ ). Therefore $\gamma_{2} \in \Gamma(2)$. Since $\gamma_{2}$ maps a point $\mathbf{y} \in \Omega$ (2) to another point $\mathbf{y}^{\prime} \in \Omega(2), \gamma_{2}$ must also be the identity transformation.

\subsubsection{Group $\mathrm{D}_{2}$}

Curves of this family, which forms a subfamily of the two-parametric family (3.6), can be represented by the equation

$$
y^{2}=z\left(z^{2}-1\right)\left(z^{2}-r^{2}\right)
$$

where $r \in \mathbb{C}$. In addition to the hyperelliptic involution, there are two more involutions: $\mu_{1}: z \rightarrow-z$ and $\mu_{2}: z \rightarrow r / z$ on the curve (3.21); the order of the full symmetry group

Fig. 4 Fundamental domain $\Omega$ of the modular group $\Gamma$

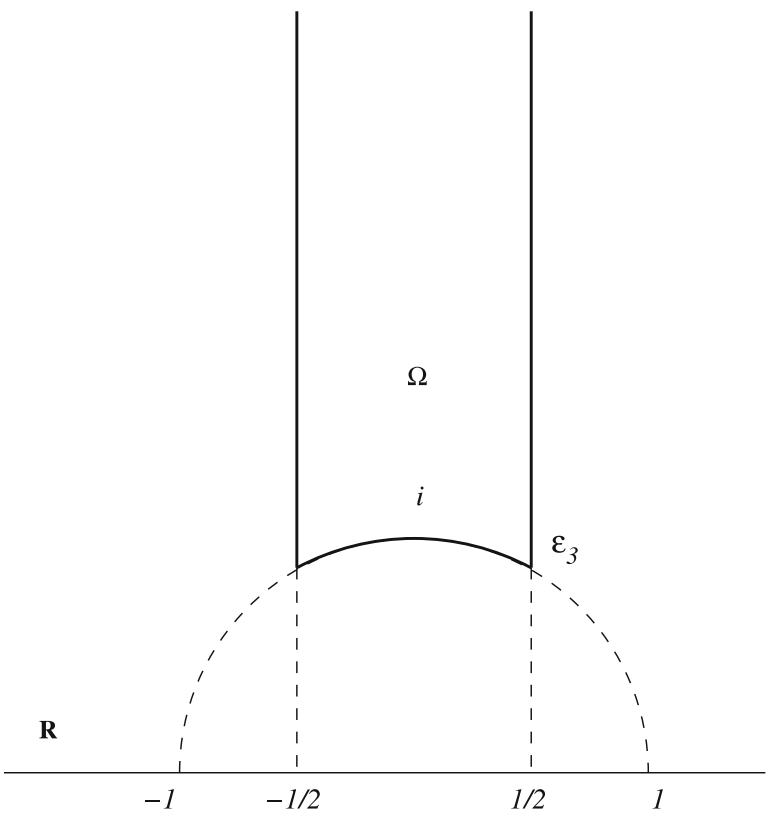




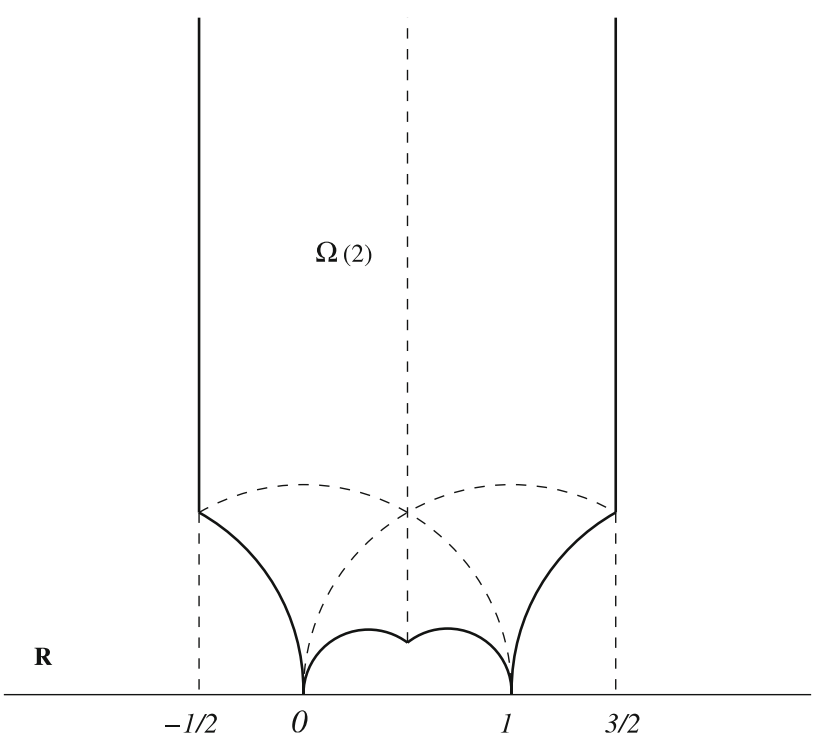

Fig. 5 Fundamental domain $\Omega(2)$ of the subgroup $\Gamma(2)$

$D_{2} \times \mathbf{Z}_{2}$ equals 8 . Choose the branch cuts and basic cycles $\left(a_{i}, b_{i}\right)$ on $\mathcal{L}$ as shown in Fig. 6 . The involutions $\mu_{1,2}$ act on this basis as follows:

$$
b_{1}^{\mu_{1}}=b_{2}-a_{1}, \quad b_{2}^{\mu_{1}}=-b_{1}+a_{2}, \quad a_{1}^{\mu_{1}}=a_{2}, \quad a_{2}^{\mu_{1}}=-a_{1},
$$

and

$$
b_{1}^{\mu_{2}}=b_{2}, \quad b_{2}^{\mu_{2}}=b_{1}, \quad a_{1}^{\mu_{2}}=a_{2}, \quad a_{2}^{\mu_{2}}=a_{1} ;
$$

therefore the action of $\mu_{1}$ and $\mu_{2}$ on the vector $\left(b_{1}, b_{2}, a_{1}, a_{2}\right)^{t}$ is given by the following $\operatorname{Sp}(4, \mathbf{Z})$ matrices:

$$
T^{\mu_{1}}=\left(\begin{array}{cccc}
0 & 1 & -1 & 0 \\
-1 & 0 & 0 & 1 \\
0 & 0 & 0 & 1 \\
0 & 0 & -1 & 0
\end{array}\right) ; \quad T^{\mu_{2}}=\left(\begin{array}{cccc}
0 & 1 & 0 & 0 \\
1 & 0 & 0 & 0 \\
0 & 0 & 0 & 1 \\
0 & 0 & 1 & 0
\end{array}\right),
$$

respectively. Invariance of the matrix of $b$-periods under the $\operatorname{Sp}(4, \mathbf{Z})$ transformation defined by $T^{\mu_{1}}$ implies the following structure of the matrix in the chosen basis:

$$
\mathbf{B}=\left(\begin{array}{cc}
\sigma & 1 / 2 \\
1 / 2 & \sigma
\end{array}\right)
$$

where $\sigma \in \mathbb{C} ; \Im \sigma>0$. Invariance of the matrix of $b$-periods under the action of $T^{\mu_{2}}$ does not impose any other restriction.

It will be convenient for us to work in terms of the parameter $\mathbf{x}=\sigma+1 / 2$, i.e., to represent the matrix $\mathbf{B}$ in the form

$$
\mathbf{B}=\left(\begin{array}{cc}
\mathbf{x}-1 / 2 & 1 / 2 \\
1 / 2 & \mathbf{x}-1 / 2
\end{array}\right)
$$




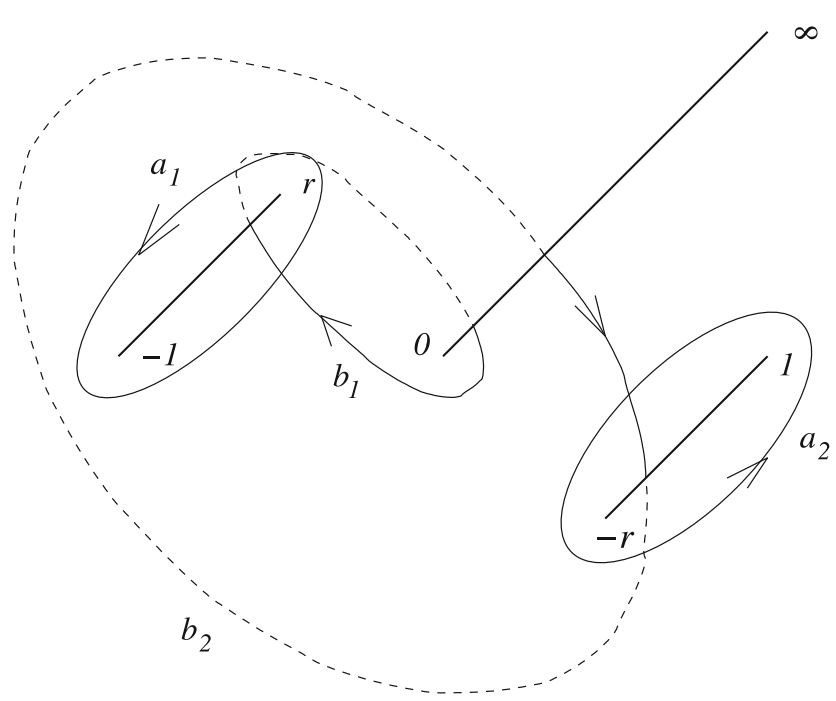

Fig. 6 Canonical basis of cycles on a curve with $D_{2} \times \mathbf{Z}_{2}$ symmetry

for $\Im \mathbf{x}>0$. The moduli space $\mathcal{M}\left(D_{2}\right)$ of $D_{2}$ curves can be realized as the upper complex half-plane in the variable $\mathbf{x}=\sigma+1 / 2$ factorized with respect to the action of the group $\operatorname{Sp}(4, \mathbf{Z})$ on matrices of the form (3.26).

The analogue of Schiller's theorem 3 for the $D_{2}$ family looks as follows. Let us introduce the modular group $\Gamma_{0}(2)$ which is the subgroup of $S L(2, \mathbf{Z})$ consisting of matrices whose (21) entry is even. The minimal subgroup of $G L(2, \mathbf{Q})$ containing $\Gamma_{0}(2)$ and the element

$$
\left(\begin{array}{cc}
0 & -1 \\
2 & 0
\end{array}\right)
$$

is called $\Gamma_{0}(2)+$. The group $\Gamma_{0}(2)+$ is generated by the following two elements $[1,7,8]$ :

$$
\gamma_{1}=\left(\begin{array}{ll}
1 & 1 \\
0 & 1
\end{array}\right) \text { and } \gamma_{2}=\left(\begin{array}{cc}
2 & -1 \\
2 & 0
\end{array}\right) .
$$

Theorem 4 The moduli space $\mathcal{M}\left(D_{2}\right)$ can be represented as the following factor:

$$
\mathcal{M}\left(D_{2}\right)=\mathcal{S}\left(D_{2}\right) / \Gamma_{0}(2)+
$$

where

$$
\mathcal{S}\left(D_{2}\right)=H \backslash\left\{\gamma\left(\frac{1}{2}+\frac{i}{2}\right), \quad \gamma \in \Gamma_{0}(2)+\right\}
$$

Proof The proof can be obtained as a corollary of Schiller's theorem 3. To restrict $\mathcal{M}\left(\mathbf{Z}_{2}\right)$ to $\mathcal{M}\left(D_{2}\right)$ one should put $\mathbf{y}=\mathbf{x}-1$. It is straightforward to verify that the subgroup of the group $G$ (3.19) preserving the constraint $\mathbf{y}=\mathbf{x}-1$ coincides with $\Gamma_{0}(2)+$.

In particular, the action of the generators $\gamma_{1}$ and $\gamma_{2}$ on $\mathbf{x}$ is equivalent to the action of the following matrices $T_{1,2} \in \mathrm{Sp}(4, \mathbf{Z})$ on the matrix (3.26), respectively:

$$
f\left(\gamma_{1}\right):=T_{1}=\left(\begin{array}{cccc}
1 & 0 & 1 & 0 \\
0 & 1 & 0 & 1 \\
0 & 0 & 1 & 0 \\
0 & 0 & 0 & 1
\end{array}\right), \quad f\left(\gamma_{2}\right):=T_{2}=\left(\begin{array}{cccc}
0 & -1 & 1 & 0 \\
0 & -1 & 0 & 0 \\
-1 & -1 & 0 & 0 \\
1 & -1 & 1 & -1
\end{array}\right) \text {. }
$$


This defines the group homomorphism $f: \Gamma_{0}(2)+\rightarrow \operatorname{Sp}(4, \mathbf{Z})$ on the generators of $\Gamma_{0}(2)+$ which extends to the whole group according to the group structure.

It remains to understand which matrices of the form (3.26) are $\operatorname{Sp}(4, \mathbf{Z})$-equivalent to diagonal ones. Since it is not obvious to get this information from Schiller's theorem 3, we choose an indirect way. First, applying the transformation

$$
\left(\begin{array}{cccc}
0 & 1 & 0 & 0 \\
0 & 1 & -1 & 0 \\
-1 & 0 & 0 & 1 \\
1 & 0 & 0 & 0
\end{array}\right)
$$

to the matrix (3.25) with $\mathbf{x}=1 / 2+i / 2$, we get the diagonal matrix $\operatorname{diag}(i, i)$. Therefore, this vertex of the fundamental domain $\Omega_{0}(2)+$ (as well as any point equivalent to this point under $\Gamma_{0}(2)+$ transformations), corresponds to a degeneration of a genus two Riemann surface to the union of two tori. On the other hand, the moduli space $\mathcal{M}\left(D_{2}\right)$ can be alternatively parametrized by $\alpha^{2}$, where $\alpha$ is the coefficient of the sextic $x y\left(x^{4}+\alpha x^{2} y^{2}+y^{4}\right)$ (this form of the equation can be obtained by a simple transformation from (3.21)); all values of $\alpha^{2}$ correspond to different points of $\mathcal{M}\left(D_{2}\right)$. In terms of $\alpha^{2}$ the space $\mathcal{M}\left(D_{2}\right)$ looks like the Riemann sphere with two deleted points: $\alpha^{2}=\infty$, and $\alpha^{2}=4$, where the Riemann surface degenerates [9]. Since we already know two degeneration points in terms of the parameter $\mathbf{x}$ ( $\mathbf{x}=i \infty$ and $\mathbf{x}=1 / 2+i / 2$, which correspond to $\alpha^{2}=\infty$ and $\alpha^{2}=4$, respectively), we can conclude that these are the only boundary points of $\mathcal{M}\left(D_{2}\right)$ in $\Omega_{0}(2)+$.

Therefore, all matrices of the form (3.25) which are $\operatorname{Sp}(4, \mathbf{Z})$-equivalent to diagonal ones, can be obtained from the value $\mathbf{x}=1 / 2+i / 2$ by a $\Gamma_{0}(2)+$ transformation.

Now we can identify the space $\mathcal{M}\left(D_{2}\right)$ with the fundamental domain $\Omega_{0}(2)+$ of the group $\Gamma_{0}(2)+$ (see Fig. 7). The vertical lines are identified by the transformation $\gamma_{1}$ from (3.27); the arcs are identified by the transformation $\gamma_{2}$ from (3.27).

The space $\mathcal{S}\left(D_{2}\right)$ (3.29) is the natural analog of the Torelli space for the $D_{2}$ family (the "special $D_{2}$ Torelli space"); the group $\Gamma_{0}(2)+$ can be naturally called the "special $D_{2}$ Torelli group", in analogy to Schiller's terminology for the case of $\mathcal{M}\left(\mathbf{Z}_{2}\right)$.

\subsubsection{Group $D_{3}$}

This is another subfamily of the two-parametric family (3.6) [4]. Curves admitting this symmetry group also form a one-parametric sub-family of (3.6); they can be represented by the equation

$$
y^{2}=\left(z^{3}-1\right)\left(z^{3}-r^{3}\right)
$$

where $r \in \mathbb{C}$. In addition to the hyperelliptic involution two more generators of the symmetry group are acting on the curve (3.31): the element of order three $\mu_{1}: z \rightarrow \epsilon_{3} z$ and the involution $\mu_{2}: z \rightarrow r / z$; the order of the full symmetry group $D_{3} \times \mathbf{Z}_{2}$ equals 12 . Choose the branch cuts and basic cycles $\left(a_{i}, b_{i}\right)$ on $\mathcal{L}$ as shown in Fig. 8. The symmetries $\mu_{1,2}$ act on this basis as follows:

$$
b_{1}^{\mu_{1}}=b_{2}-b_{1}, \quad b_{2}^{\mu_{1}}=-b_{1}, \quad a_{1}^{\mu_{1}}=a_{2}, \quad a_{2}^{\mu_{1}}=-a_{1}-a_{2} ;
$$

and

$$
b_{1}^{\mu_{2}}=b_{1}-b_{2}, \quad b_{2}^{\mu_{2}}=-b_{2}, \quad a_{1}^{\mu_{2}}=a_{1}, \quad a_{2}^{\mu_{2}}=-a_{1}-a_{2} ;
$$




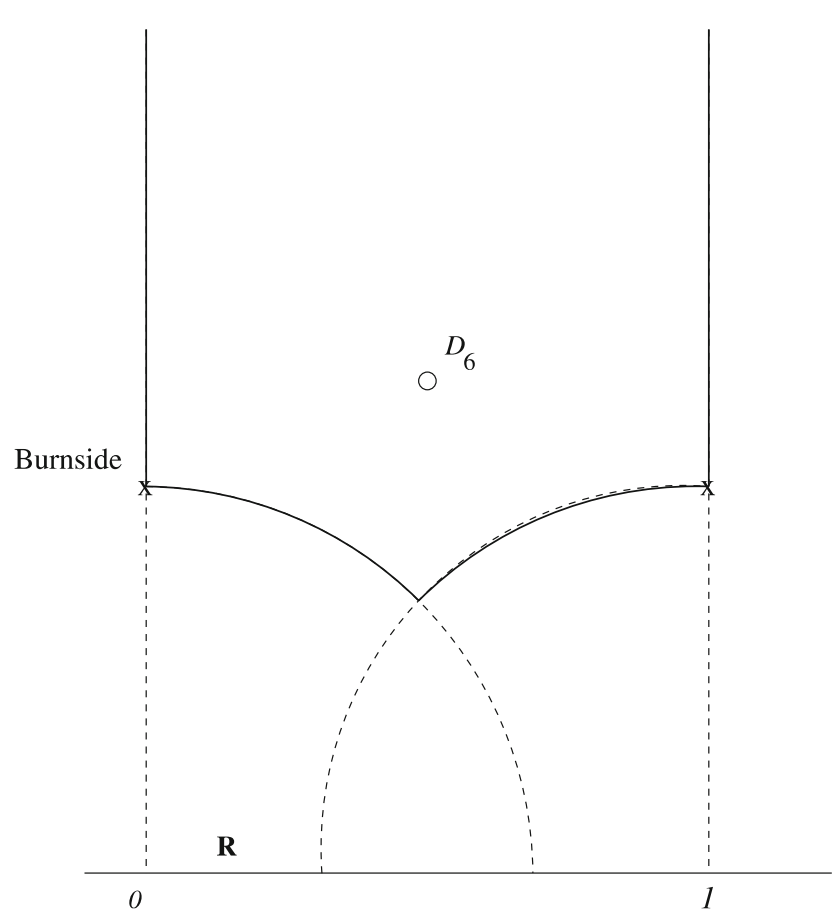

Fig. 7 Fundamental domain $\Omega_{0}(2)+$ with critical points of $\mathcal{F}$. The bounding circles have radius $1 / \sqrt{2}$ and centers at 0 and 1 . The Burnside curve corresponds to two values of $\sigma$ marked by $\mathrm{x}$; the point marked by a small circle corresponds to the $D_{6}$ curve

therefore the action of $\mu_{1}$ and $\mu_{2}$ on the vector $\left(b_{1}, b_{2}, a_{1}, a_{2}\right)^{t}$ is given by the following $\operatorname{Sp}(4, \mathbf{Z})$ matrices:

$$
T^{\mu_{1}}=\left(\begin{array}{cccc}
-1 & 1 & 0 & 0 \\
-1 & 0 & 0 & 0 \\
0 & 0 & 0 & 1 \\
0 & 0 & -1 & -1
\end{array}\right) ; \quad T^{\mu_{2}}=\left(\begin{array}{cccc}
1 & -1 & 0 & 0 \\
0 & -1 & 0 & 0 \\
0 & 0 & 1 & 0 \\
0 & 0 & -1 & -1
\end{array}\right),
$$

respectively.

Invariance of the matrix of $b$-periods under the transformation defined by $T^{\mu_{1}}$ implies its following structure in the chosen basis:

$$
\mathbf{B}=\left(\begin{array}{cc}
2 \sigma & \sigma \\
\sigma & 2 \sigma
\end{array}\right)
$$

where $\sigma \in \mathbb{C}$; $\Im \sigma>0$. Invariance of the matrix of $b$-periods matrix under the action of $T^{\mu_{2}}$ does not impose any other restriction.

Introduce the subgroup $\Gamma_{0}(3)$ of $S L(2, \mathbf{Z})$, which consists of matrices whose (21) matrix entry $\equiv 0(\bmod 3)$. The minimal subgroup of $G L(2, \mathbf{Q})$ containing both $\Gamma_{0}(3)$ and the matrix

$$
\left(\begin{array}{cc}
0 & -1 \\
3 & 0
\end{array}\right)
$$




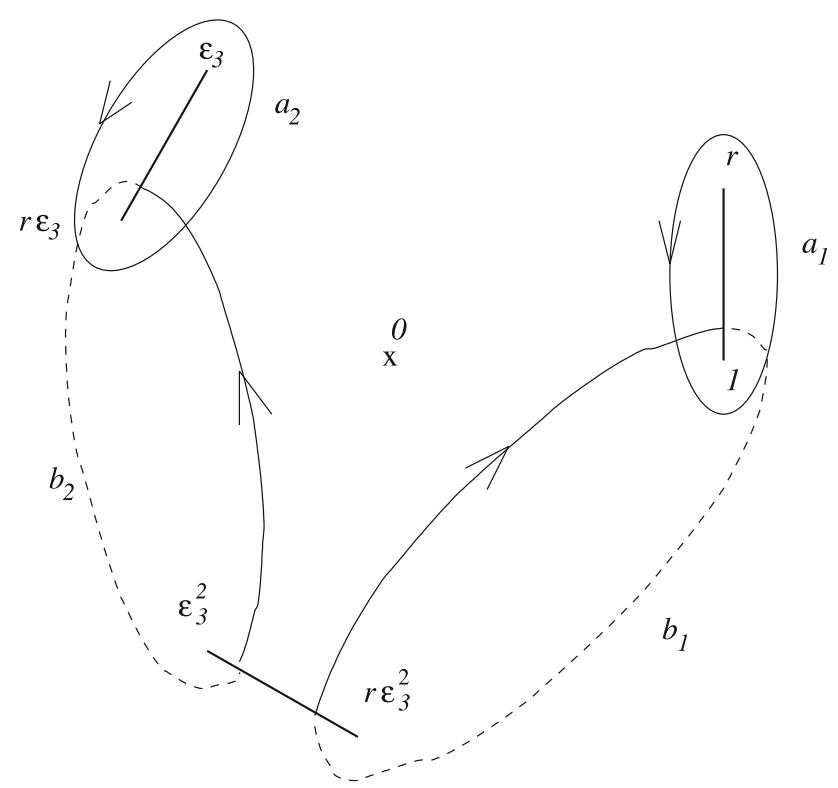

Fig. 8 Canonical basis of cycles on a curve with $D_{2} \times \mathbf{Z}_{2}$ symmetry

is called $\Gamma_{0}(3)+$. The group $\Gamma_{0}(3)+$ is generated by the following two elements:

$$
\gamma_{1}=\left(\begin{array}{ll}
1 & 1 \\
0 & 1
\end{array}\right), \quad \gamma_{2}=\left(\begin{array}{cc}
3 & -1 \\
3 & 0
\end{array}\right)
$$

Theorem 5 The moduli space $\mathcal{M}\left(D_{3}\right)$ can be represented as the following factor:

$$
\mathcal{M}\left(D_{3}\right)=\mathcal{S}\left(D_{3}\right) / \Gamma_{0}(3)+
$$

where

$$
\mathcal{S}\left(D_{3}\right)=H \backslash\left\{\gamma\left(\frac{1}{2}+\frac{i}{2 \sqrt{3}}\right), \gamma \in \Gamma_{0}(3)+\right\}
$$

Proof The proof can be obtained as a corollary of Schiller's Theorem 3. To restrict $\mathcal{M}\left(\mathbf{Z}_{2}\right)$ to $\mathcal{M}\left(D_{3}\right)$ one should put $\mathbf{x}=3 \sigma, \mathbf{y}=\sigma$. It is straightforward to verify that the subgroup of the group $G$ (3.19) preserving the constraint $\mathbf{x}=3 \mathbf{y}$ coincides with $\Gamma_{0}(3)+$ acting on $\sigma(\equiv \mathbf{y})$.

In particular, the action of the generators $\gamma_{1}$ and $\gamma_{2}$ on $\sigma$ is equivalent to the action of the following $\operatorname{Sp}(4, \mathbf{Z})$ matrices $T_{1,2} \in \mathrm{Sp}(4, \mathbf{Z})$ on the matrix of $b$-periods (3.35), respectively:

$$
f\left(\gamma_{1}\right):=T_{1}=\left(\begin{array}{cccc}
1 & 0 & 2 & 1 \\
0 & 1 & 1 & 2 \\
0 & 0 & 1 & 0 \\
0 & 0 & 0 & 1
\end{array}\right), \quad f\left(\gamma_{2}\right):=T_{2}=\left(\begin{array}{cccc}
-2 & 1 & 1 & 0 \\
-1 & 2 & 0 & -1 \\
-1 & 0 & 0 & 0 \\
0 & 1 & 0 & 0
\end{array}\right)
$$

This defines the group homomorphism $f: \Gamma_{0}(3)+\rightarrow \operatorname{Sp}(4, \mathbf{Z})$ on the generators of $\Gamma_{0}(3)+$ which extends to the whole group according to the group structure. 
It remains to understand which matrices of the form (3.35) are $\operatorname{Sp}(4, \mathbf{Z})$ - equivalent to diagonal ones. Again, it is not obvious to get this information from Schiller's theorem 3, and we shall use well-known facts about the structure of $\mathcal{M}\left(D_{3}\right)$ in terms of the coefficients of the sextic.

First, applying the transformation

$$
\left(\begin{array}{cccc}
0 & 1 & 0 & 0 \\
0 & 1 & -1 & 0 \\
-1 & 0 & 0 & 1 \\
1 & 0 & 0 & 0
\end{array}\right)
$$

to the matrix (3.35) with $\mathbf{x}=1 / 2+i / 2 \sqrt{3}$, we get the diagonal matrix $\operatorname{diag}\left(-\frac{1}{2}+\frac{i \sqrt{3}}{2}, \frac{1}{2}+\right.$ $\frac{i \sqrt{3}}{2}$ ). Therefore, this point (as well as any point equivalent to this point under $\Gamma_{0}(3)+$ transformations), corresponds to a degeneration of a genus two Riemann surface to the union of two tori.

On the other hand, the moduli space $\mathcal{M}\left(D_{3}\right)$ can be alternatively parametrized by $\alpha^{2}$, where $\alpha$ is the coefficient of the sextic $x^{6}+\alpha x^{3} y^{3}-y^{6}$ (this form of the equation can be obtained by a simple transformation from (3.31)); all values of $\alpha^{2}$ correspond to different points of $\mathcal{M}\left(D_{2}\right)$. In terms of $\alpha^{2}$ the space $\mathcal{M}\left(D_{3}\right)$ looks like the Riemann sphere with two deleted points: $\alpha^{2}=\infty$, and $\alpha^{2}=-4$, where the Riemann surface degenerates [9]. Since we already know two degeneration points in terms of the parameter $\mathbf{x}(\mathbf{x}=i \infty$ and $\mathbf{x}=1 / 2+i / 2 \sqrt{3}$, which correspond to $\alpha^{2}=\infty$ and $\alpha^{2}=-4$, respectively), we can conclude that these are the only boundary points of $\mathcal{M}\left(D_{3}\right)$ in $\Omega_{0}(3)+$.

Therefore, all matrices of the form (3.35) which are $\operatorname{Sp}(4, \mathbf{Z})$ - equivalent to a diagonal one, can be obtained from the value $\mathbf{x}=1 / 2+i / 2 \sqrt{3}$ by a $\Gamma_{0}(3)+$ transformation.

Now we can identify the space $\mathcal{M}\left(D_{3}\right)$ with the fundamental domain $\Omega_{0}(3)+$ of the group $\Gamma_{0}(3)+$ (see Fig. 9). The vertical lines are identified by the transformation $\gamma_{1}$ from (3.36); the arcs are identified by the transformation $\gamma_{2}$ from (3.36).

The space $\mathcal{S}\left(D_{3}\right)$ (3.38) is the natural analog of the Torelli space for the $D_{3}$ family (the "special $D_{3}$ Torelli space"); the group $\Gamma_{0}(3)+$ can be naturally called the "special $D_{3}$ Torelli group".

\subsubsection{Group $S_{4}$}

The curve admitting this reduced symmetry group is defined by the equation

$$
y^{2}=z\left(z^{4}-1\right)
$$

it is called the Burnside curve. In addition to the hyperelliptic involution the generators of the symmetry group of the Burnside curve are given by $\mu_{1}: z \rightarrow i z, \mu_{2}: z \rightarrow(z+1) /(z-1)$ and $\mu_{3}: z \rightarrow-\frac{1}{z}$. The order of the full symmetry group $S_{4} \times \mathbf{Z}_{2}$ of the Burnside curve equals 48. The Burnside curve belongs to both the $D_{2}$ and the $D_{3}$ families.

Choose the branch cuts and basic cycles $\left(a_{i}, b_{i}\right)$ on $\mathcal{L}$ as shown in Fig. 6 for $r=i$. The generators $\mu_{1,2,3}$ act on this basis as follows:

$$
\begin{aligned}
& b_{1}^{\mu_{1}}=-b_{2}+a_{1}-a_{2}, \quad b_{2}^{\mu_{1}}=b_{2}+a_{2}, \quad a_{1}^{\mu_{1}}=-b_{1}+b_{2}-a_{1}+a_{2}, \quad a_{2}^{\mu_{1}}=-b_{1}-b_{2}, \\
& b_{1}^{\mu_{2}}=-b_{1}, \quad b_{2}^{\mu_{2}}=b_{1}+b_{2}, \quad a_{1}^{\mu_{2}}=b_{2}-a_{1}+a_{2}, \quad a_{2}^{\mu_{2}}=-b_{1}+a_{2} ;
\end{aligned}
$$




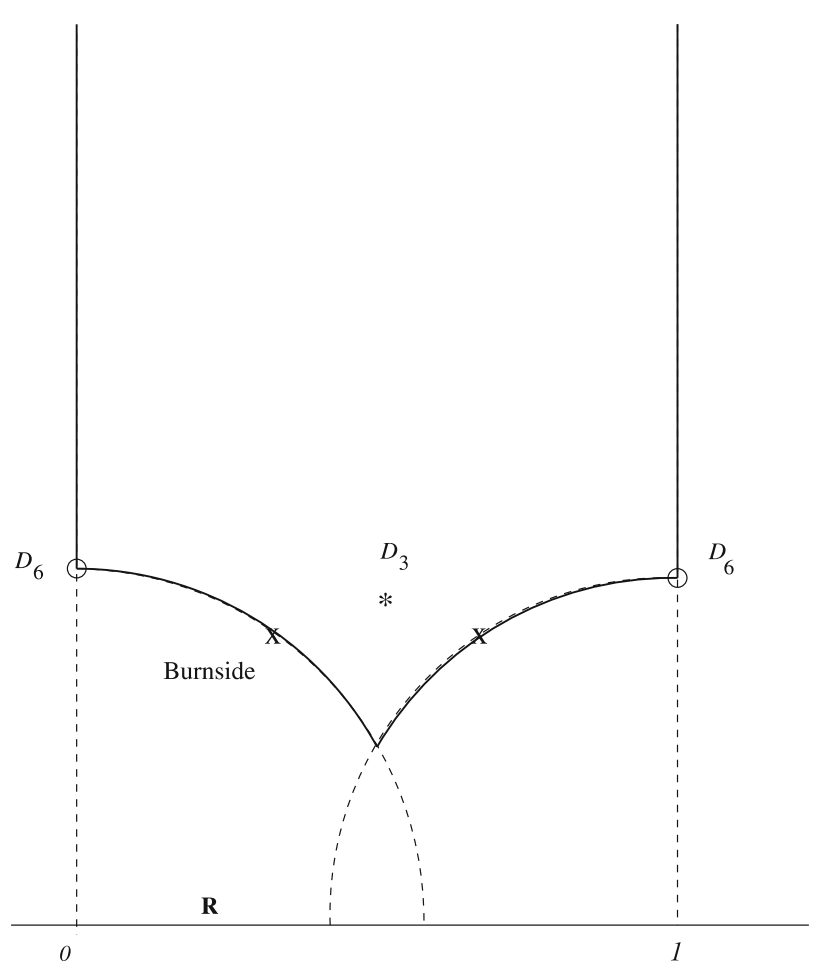

Fig. 9 Fundamental domain $\Omega_{0}(3)+$. The bounding circles have radius $1 / \sqrt{3}$ and centers at 0 and 1 . The small circles mark the $D_{6}$ curve, $\mathrm{x}$ denotes the Burnside curve, and $*$ denotes the critical $D_{3}$ curve

and

$$
b_{1}^{\mu_{3}}=b_{2}, \quad b_{2}^{\mu_{3}}=b_{1}, \quad a_{1}^{\mu_{3}}=a_{2}, \quad a_{2}^{\mu_{3}}=a_{1} ;
$$

therefore the action of $\mu_{1}, \mu_{2}$ and $\mu_{3}$ on the vector $\left(b_{1}, b_{2}, a_{1}, a_{2}\right)^{t}$ is given by the following matrices:

$$
T^{\mu_{1}}=\left(\begin{array}{cccc}
0 & -1 & 1 & -1 \\
0 & 1 & 0 & 1 \\
-1 & 1 & -1 & 1 \\
-1 & -1 & 0 & 0
\end{array}\right), T^{\mu_{2}}=\left(\begin{array}{cccc}
-1 & 0 & 0 & 0 \\
1 & 1 & 0 & 0 \\
0 & 1 & -1 & 1 \\
-1 & 0 & 0 & 1
\end{array}\right), T^{\mu_{3}}=\left(\begin{array}{cccc}
0 & 1 & 0 & 0 \\
1 & 0 & 0 & 0 \\
0 & 0 & 0 & 1 \\
0 & 0 & 1 & 0
\end{array}\right),
$$

respectively. Invariance of the matrix of $b$-periods under the transformations defined by $T^{\mu_{1}}$ and $T^{\mu_{3}}$ implies the following structure of the matrix in the chosen basis:

$$
\mathbf{B}=\left(\begin{array}{ccc}
-\frac{1}{2}+\frac{i}{\sqrt{2}} & \frac{1}{2} \\
\frac{1}{2} & -\frac{1}{2}+\frac{i}{\sqrt{2}}
\end{array}\right)
$$

this matrix is also invariant under the transformation defined by $T^{\mu_{2}}$. 


\subsubsection{Group $D_{6}$}

The curve admitting this reduced symmetry group is defined by the equation

$$
y^{2}=z^{6}-1 \text {. }
$$

In addition to the hyperelliptic involution, there are two more independent generators of the symmetry group acting on the curve (3.45): the element of order six $\mu_{1}: z \rightarrow \epsilon_{6} z$ and the involution $\mu_{2}: z \rightarrow-1 / z$. The order of the full symmetry group $D_{6} \times \mathbf{Z}_{2}$ equals 24 . This curve belongs to both the $D_{2}$ and $D_{3}$ families.

Choose the branch cuts and basic cycles $\left(a_{i}, b_{i}\right)$ on $\mathcal{L}$ as shown in Fig. 8 for $r=\epsilon_{6}$. The involutions $\mu_{1,2}$ act on this basis as follows:

$$
b_{1}^{\mu_{1}}=-a_{1}, \quad b_{2}^{\mu_{1}}=-a_{1}-a_{2}, \quad a_{1}^{\mu_{1}}=b_{1}-b_{2}, \quad a_{2}^{\mu_{1}}=b_{2},
$$

and

$$
b_{1}^{\mu_{2}}=b_{2}, \quad b_{2}^{\mu_{2}}=b_{1}, \quad a_{1}^{\mu_{2}}=a_{2}, \quad a_{2}^{\mu_{2}}=a_{1} ;
$$

therefore the vector $\left(b_{1}, b_{2}, a_{1}, a_{2}\right)^{t}$ transforms under the action of $\mu_{1}$ and $\mu_{2}$ by the following $\operatorname{Sp}(4, \mathbf{Z})$ matrices:

$$
T^{\mu_{1}}=\left(\begin{array}{cccc}
0 & 0 & -1 & 0 \\
0 & 0 & -1 & -1 \\
1 & -1 & 0 & 0 \\
0 & 1 & 0 & 0
\end{array}\right) ; \quad T^{\mu_{2}}=\left(\begin{array}{cccc}
0 & 1 & 0 & 0 \\
1 & 0 & 0 & 0 \\
0 & 0 & 0 & 1 \\
0 & 0 & 1 & 0
\end{array}\right),
$$

respectively. Invariance of the matrix of $b$-periods under transformations defined by $T^{\mu_{1}}$ and $T^{\mu_{2}}$ implies the following structure of the matrix in the chosen basis:

$$
\mathbf{B}=\frac{i}{\sqrt{3}}\left(\begin{array}{ll}
2 & 1 \\
1 & 2
\end{array}\right) .
$$

\subsubsection{Group $\mathbf{Z}_{5}$}

The curve admitting this reduced symmetry group is defined by the equation

$$
y^{2}=z^{5}-1
$$

The generator of the $\mathbf{Z}_{5}$ symmetry is given by $\mu: z \rightarrow \epsilon_{5} z$. The order of the full symmetry group $\mathbf{Z}_{5} \times \mathbf{Z}_{2}$ equals 10 . This curve does not belong to any family of symmetric curves mentioned above. Choose the branch cuts and basic cycles $\left(a_{i}, b_{i}\right)$ on $\mathcal{L}$ as shown in Fig. 10. The generator $\mu$ acts on this basis as follows:

$$
b_{1}^{\mu}=-b_{1}+b_{2}, \quad b_{2}^{\mu}=-b_{1}+a_{1}+a_{2}, \quad a_{1}^{\mu}=a_{2}, \quad a_{2}^{\mu}=-b_{1},
$$

therefore the action of the generator $\mu$ on the vector $\left(b_{1}, b_{2}, a_{1}, a_{2}\right)^{t}$ is given by the following $\operatorname{Sp}(4, \mathbf{Z})$ matrix:

$$
T^{\mu}=\left(\begin{array}{cccc}
-1 & 1 & 0 & 0 \\
-1 & 0 & 1 & 1 \\
0 & 0 & 0 & 1 \\
-1 & 0 & 0 & 0
\end{array}\right)
$$




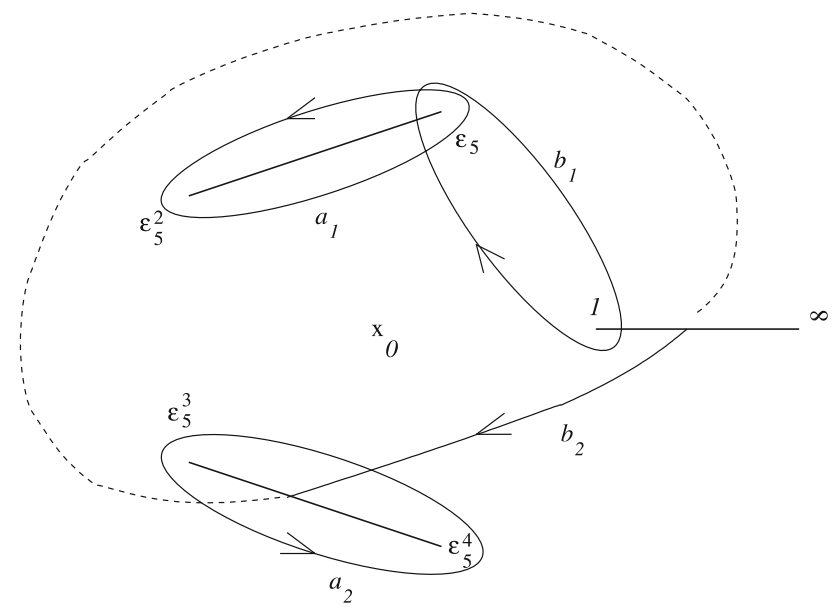

Fig. 10 Canonical basis of cycles on the curve with $\mathbf{Z}_{5} \times \mathbf{Z}_{2}$ symmetry

Invariance of the matrix of $b$-periods under the transformation defined by $T^{\mu}$ implies together with the positive definiteness of the matrix $\mathfrak{\Im} \mathbf{B}$ the following structure of this matrix in the chosen basis:

$$
\mathbf{B}=\left(\begin{array}{cc}
\epsilon_{5} & \epsilon_{5} /\left(1+\epsilon_{5}\right) \\
\epsilon_{5} /\left(1+\epsilon_{5}\right) & 1-\epsilon_{5}^{4}
\end{array}\right) .
$$

The curves (3.45), (3.39), (3.50) are curves with large automorphism groups, i.e., they do not admit small deformations preserving their groups of symmetries. They play an important role in the subsequent analysis.

\section{$4 \mathcal{F}$ on the full moduli space of genus two curves}

\subsection{Boundary behavior and symmetry of $\mathcal{F}$}

Consider $\mathcal{F}(1.1)$ as a function on Gottschling's fundamental domain $\mathcal{G}$. It is straightforward to prove the following lemma:

Lemma 3 The function $\mathcal{F}$ (1.1) vanishes if $\mathbf{B} \in \mathcal{D}$, as well as if $\mathbf{B}$ tends to the infinity of $\mathcal{G}$.

Proof (see, for example [33]; we reproduce the proof here for completeness) The definition of the function of zero argument with vectors of characteristics $\mathbf{p}, \mathbf{q}$, where $\mathbf{p}=\left(p_{1}, p_{2}\right)$ and $\mathbf{q}=\left(q_{1}, q_{2}\right)$, reads

$$
\Theta\left[\begin{array}{l}
\mathbf{p} \\
\mathbf{q}
\end{array}\right](0 \mid \mathbf{B})=\sum_{\mathbf{m} \in \mathbf{Z}^{2}} \exp \{\pi i\langle\mathbf{B}(\mathbf{m}+\mathbf{p}), \mathbf{m}+\mathbf{p}\rangle+2 \pi i\langle\mathbf{m}+\mathbf{p}, \mathbf{q}\rangle\}
$$

Suppose that $\mathbf{B} \in \mathcal{D}$; taking into account the modular invariance of $\mathcal{F}$, we can assume $\mathbf{B}_{12}=0$, while $\mathbf{B}_{11}$ and $\mathbf{B}_{22}$ remain finite. Then the genus two theta function splits into the product of two genus one theta functions with moduli $\mathbf{B}_{11}$ and $\mathbf{B}_{22}$ :

$$
\Theta\left[\begin{array}{l}
\mathbf{p} \\
\mathbf{q}
\end{array}\right](0 \mid \mathbf{B})=\vartheta\left[\begin{array}{c}
p_{1} \\
q_{1}
\end{array}\right]\left(0 \mid \mathbf{B}_{11}\right) \vartheta\left[\begin{array}{c}
p_{2} \\
q_{2}
\end{array}\right]\left(0 \mid \mathbf{B}_{22}\right)
$$


Therefore the even genus two theta constant corresponding to $\mathbf{p}=\mathbf{q}=(1 / 2,1 / 2)$ vanishes in this limit $\left(\vartheta\left[\begin{array}{l}1 / 2 \\ 1 / 2\end{array}\right]\left(0 \mid \mathbf{B}_{11}\right)=0\right.$ since $\left[\begin{array}{l}1 / 2 \\ 1 / 2\end{array}\right]$ is the odd genus one characteristic $)$.

Now consider the part of the boundary of $\mathcal{M}$, where the matrix of $b$-periods diverges, i.e., where some or all $y_{i}$ from (3.1) tend to $+\infty$. Then $\operatorname{det} \Im \mathbf{B}$ diverges as a polynomial (of degree 2 with respect to $y_{2}$ and degree 1 with respect to $y_{1}$ and $y_{3}$ ), while some theta constants vanish exponentially.

To simplify the numerical analysis of the behavior of the function $\mathcal{F}$ on $\mathcal{G}$ we shall take into account the symmetry provided by the following lemma:

Lemma 4 The function $\mathcal{F}$ has the following symmetry property:

$$
\mathcal{F}(-\overline{\mathbf{B}})=\mathcal{F}(\mathbf{B})
$$

Proof This is a straightforward analog of the symmetry (2.2) in the genus one case. The proof is simple: each theta constant satisfies the relation $\Theta\left[\beta_{s}\right](-\overline{\mathbf{B}})=\overline{\Theta\left[\beta_{s}\right](\mathbf{B})}$ as a corollary of the definition (4.1); together with an obvious symmetry of $\operatorname{det} \Im \mathbf{B}$ this implies (4.3).

\subsection{Curves with large automorphism groups as critical points of $\mathcal{F}$}

Here we show that all three Riemann surfaces $\left(D_{6}\right.$, Burnside and $\left.\mathbf{Z}_{5}\right)$ with large groups of automorphisms are critical points of any $\operatorname{Sp}(4, \mathbf{Z})$ invariant function on $\mathcal{H}$ which is realanalytic in open neighborhoods of the matrices of $b$-periods of these curves. In particular these Riemann surfaces are critical points of our function $\mathcal{F}(1.1)$.

Consider an arbitrary (real-)analytic function $F$ on $\mathcal{H} \backslash \mathcal{D}$. To analyze the power series of the function $F$ in a neighborhood of some point of $\mathcal{H}$, it is convenient to map $\mathcal{H}$ to the generalized unit ball

$$
\mathfrak{U}=\left\{w \in M(2, \mathbb{C}): w=w^{t} ; w w^{*} \leq I\right\}
$$

in a way that the center of the power series is mapped to the origin. Consider the Cayley transformation

$$
z \mapsto w=(z-i I)(z+i I)^{-1},
$$

where $I$ is the $2 \times 2$ unit matrix, which gives a biholomorphic map $\mathcal{H} \rightarrow \mathfrak{U}$.

To map a given point of $\mathcal{H}$ to the origin of $\mathfrak{U}$ we shall superpose the Cayley transformation with some automorphism of $\mathfrak{U}$. Recall (see [32]) that all holomorphic automorphisms of the generalized unit ball $\mathfrak{U}$ are given by the transformations

$$
w \mapsto(A w+B)(C w+D)^{-1},
$$

where the $(2 \times 2)$-matrices $A, B, C, D$ satisfy the following constraints:

$$
C=\bar{B}, \quad D=\bar{A}, \quad \bar{A} A^{t}-\bar{B} B^{t}=I, \quad A B^{t}=B A^{t} .
$$

A holomorphic transformation of $\mathfrak{U}$ to itself such that a given point $S \in \mathfrak{U}$ is mapped to the origin, looks as follows (see [32, p. 177]). Introduce an auxiliary matrix

$$
R=\sqrt{I_{2}-S \bar{S}}
$$

and define matrices $A, B, C, D$ by

$$
A=\overline{R^{-1}}, \quad B=-A S, \quad C=\bar{B}, \quad D=\bar{A} .
$$


These matrices satisfy (4.5) and

$$
(A S+B)(C S+D)^{-1}=0 .
$$

Consider a point $z_{0}$ of the Siegel half-space $\mathcal{H}$ and map it to the point $S=\left(z_{0}-i I_{2}\right)\left(z_{0}+\right.$ $\left.i I_{2}\right)^{-1} \in \mathfrak{U}$. Define matrices $A_{1}, B_{1}, C_{1}, D_{1}$ by the equality

$$
\left(\begin{array}{ll}
A_{1} & B_{1} \\
C_{1} & D_{1}
\end{array}\right)=\left(\begin{array}{ll}
A & B \\
C & D
\end{array}\right)\left(\begin{array}{cc}
I_{2} & -i I_{2} \\
I_{2} & i I_{2}
\end{array}\right)
$$

with $A, B, C, D$ from (4.7).

Then the biholomorphic transformation $\mathcal{K}_{z_{0}}$ between the upper Siegel half-space and the generalized unit ball $\mathcal{K}_{z_{0}}: \mathcal{H} \rightarrow \mathfrak{U}$ defined by

$$
\mathcal{K}_{z_{0}}(z)=\left(A_{1} z+B_{1}\right)\left(C_{1} z+D_{1}\right)^{-1},
$$

maps the point $z_{0} \in \mathcal{H}$ to $0 \in \mathfrak{U}$.

Theorem 6 Let $F$ be any $\operatorname{Sp}(4, \mathbf{Z})$-invariant function on $\mathcal{H}$, which is $C^{1}$ in open neighborhoods of the points

$$
\mathbf{B}_{1}=\left(\begin{array}{cc}
\frac{i}{\sqrt{2}}-\frac{1}{2} & \frac{1}{2} \\
\frac{1}{2} & \frac{i}{\sqrt{2}}-\frac{1}{2}
\end{array}\right), \quad \mathbf{B}_{2}=\frac{i}{\sqrt{3}}\left(\begin{array}{ll}
2 & 1 \\
1 & 2
\end{array}\right) \quad \text { and } \quad \mathbf{B}_{3}=\left(\begin{array}{cc}
\epsilon_{5} & \frac{\epsilon_{5}}{1+\epsilon_{5}} \\
\frac{\epsilon_{5}}{1+\epsilon_{5}} & 1-\epsilon_{5}^{4}
\end{array}\right)
$$

which are the matrices of b-periods of the curves with large automorphism groups $y=$ $x\left(x^{4}-1\right), y=x^{6}-1$ and $y=z^{5}-1$ respectively. Then $\operatorname{grad} F$ vanishes at the points $\mathbf{B}_{1}$, $\mathbf{B}_{2}$ and $\mathbf{B}_{3}$.

Proof 1. Point $\mathbf{B}_{1}$. Let $T:=T^{\mu_{1}}$ where $T^{\mu_{1}}$ is the first of the matrices (3.43) which leave the point $\mathbf{B}_{1}$ invariant.

The function $g: \mathfrak{U} \rightarrow \mathbb{C}$ defined by

$$
g(w):=F\left(\mathcal{K}_{\mathbf{B}_{1}}^{-1} w\right)
$$

satisfies the equation

$$
g(w):=g\left(\mathcal{K}_{\mathbf{B}_{1}} T \mathcal{K}_{\mathbf{B}_{1}}^{-1} w\right)
$$

One can check that

$$
\mathcal{K}_{\mathbf{B}_{1}} T \mathcal{K}_{\mathbf{B}_{1}}^{-1} w=U_{1} w U_{1}^{t}
$$

with a unitary matrix $U_{1}$ (recall that a $4 \times 4$ matrix $\mathcal{K}_{\mathbf{B}_{1}} T \mathcal{K}_{\mathbf{B}_{1}}^{-1}$ having the block structure

$$
\left(\begin{array}{ll}
A & B \\
C & D
\end{array}\right)
$$

acts on a $2 \times 2$ matrix $w$ according to (4.4)). Let

$$
w=\left(\begin{array}{ll}
x & z \\
z & y
\end{array}\right), \quad U_{1}=\left(\begin{array}{cc}
u & v \\
w & t
\end{array}\right) \quad \text { and } \quad U_{1} w U_{1}^{t}=\left(\begin{array}{cc}
\tilde{x} & \tilde{z} \\
\tilde{z} & \tilde{y}
\end{array}\right)
$$

then

$$
\left(\begin{array}{c}
\tilde{x} \\
\tilde{y} \\
\tilde{z}
\end{array}\right)=\mathbb{A}\left(\begin{array}{l}
x \\
y \\
z
\end{array}\right)
$$


with

$$
\mathbb{A}=\left(\begin{array}{ccc}
u^{2} & v^{2} & 2 u v \\
w^{2} & t^{2} & 2 t w \\
u w & v t & v w+u t
\end{array}\right)
$$

The eigenvalues of the matrix $\mathbb{A}$ are given by $\{-1, i,-i\}$.

Due to (4.10) the partial derivatives $g_{x}, g_{y}$ and $g_{z}$ of the function $g$ at the point $w=0$ satisfy the equation

$$
\left(\begin{array}{l}
g_{x} \\
g_{y} \\
g_{z}
\end{array}\right)=\mathbb{A}^{t}\left(\begin{array}{l}
g_{x} \\
g_{y} \\
g_{z}
\end{array}\right) .
$$

Since 1 is not an eigenvalue of the matrix $\mathbb{A}$, all these derivatives vanish, as well as all derivatives of the function $F$.

2. Point $\mathbf{B}_{2}$. Here the same scheme applies to the matrix $T^{\mu_{1}}$ from (3.48), which belongs to the stabilizer of the point $\mathbf{B}_{2}$. In this case the spectrum of the corresponding matrix $\mathbb{A}$ looks as follows:

$$
\operatorname{spectrum}(\mathbb{A})=\left\{-1, \epsilon_{3}, \epsilon_{3}^{2}\right\} ;
$$

since again 1 is not in the spectrum, $\operatorname{grad} g$ vanishes at the origin and $\operatorname{grad} F \operatorname{vanishes}$ at $\mathbf{B}_{2}$.

3. Point $\mathbf{B}_{3}$. In this case we use the modular transformation (3.52) which leaves the point $\mathbf{B}_{3}$ invariant. Then the spectrum of the corresponding matrix $\mathbb{A}$ reads:

$$
\operatorname{spectrum}(\mathbb{A})=\left(\epsilon_{5}, \epsilon_{5}^{2}, \epsilon_{5}^{4}\right)
$$

since again 1 is not among the eigenvalues, $\operatorname{grad} g$ vanishes at the origin. Thus $\operatorname{grad} F$ vanishes at $\mathbf{B}_{3}$.

The Theorem 6 applies to our function $\mathcal{F}(1.1)$ due to the following

Lemma 5 The function $\mathcal{F}$, defined by (1.1), satisfies the conditions of theorem 6.

The non-trivial part of the proof of this lemma is the verification of the fact that the function $\mathcal{F}(1.1)$ is invariant under $\operatorname{Sp}(4, \mathbf{Z})$ transformations of matrix of $b$-periods. This invariance can be easily verified directly, using the transformation law of theta-constants under these transformations of the matrix of $b$-periods. Alternatively, this invariance follows from the link of $\mathcal{F}$ and the determinant of the Laplacian in the Bergman metric (1.2), taking into account that the determinant is independent of the choice of a canonical basis of cycles on $\mathcal{L}$.

\subsection{Numerical results}

The rigorous results established above can be summarized as follows: the function $\mathcal{F}$ vanishes at the boundary of $\mathcal{M}$ and has at least three critical points corresponding to Riemann surfaces with large automorphism groups. As we shall see below, knowing the value of the virtual Euler characteristic of $\mathcal{M}$, one can prove the existence of another critical point of $\mathcal{F}$.

However, since it was only possible to completely characterize the extremal points of even genus 1 modular functions numerically (see Sect. 2), there is little hope for the time being to obtain complete analytical results for the decisively more complicated case of genus 2 . Thus we turn to a numerical approach to search for critical points of $\mathcal{F}$ in $\mathcal{G}$. 
The unboundedness of the domain $\mathcal{G}$ in the directions of $y_{1}, y_{2}$ and $y_{3}(3.1)$ is not a problem since the function $\mathcal{F}$ decreases exponentially for large $y_{i}$. It turns out that a restriction to value of $y_{i}$ with $y_{i} \leq 2$ within the Gottschling domain is sufficient (notice that the algorithm explained below finds critical points with bigger values of the $y_{i}$, but these do not lie in the fundamental domain). Furthermore we use the symmetry (4.3) which in terms of $\left\{x_{i}, y_{i}\right\}$ looks as follows: $\mathcal{F}\left(\left\{-x_{i}, y_{i}\right\}\right)=\mathcal{F}\left(\left\{x_{i}, y_{i}\right\}\right)$ to decrease the amount of computation by a factor of 2: in addition to Gottschling's conditions we assume $x_{3}>0$.

To locate the critical points of $\mathcal{F}$ inside of $\mathcal{G}$ we need to analyze the length of $\operatorname{grad} \mathcal{F}$ in $\mathcal{G}$. For the numerical evaluation of $\operatorname{grad} \mathcal{F}$ at a given point of $\mathcal{M}$, we first differentiate $\mathcal{F}$ analytically with respect to the $\mathbf{B}_{i j}$. The differentiation of $\operatorname{det} \Im \mathbf{B}$ is obvious; to differentiate theta constants we differentiate term by term the series (4.1) to get:

$$
\begin{aligned}
\frac{\partial}{\partial \mathbf{B}_{j k}} \Theta\left[\begin{array}{l}
\mathbf{p} \\
\mathbf{q}
\end{array}\right](0 \mid \mathbf{B})= & \sum_{m_{1}, m_{2} \in \mathbf{Z}} 2 \pi i\left(m_{j}+p_{j}\right)\left(m_{k}+p_{k}\right) /\left(1+\delta_{j k}\right) \\
& \times \exp \left\{\pi i \sum_{l, n=1,2} \mathbf{B}_{l n}\left(m_{l}+p_{l}\right)\left(m_{n}+p_{n}\right)+2 \pi i \sum_{l=1,2}\left(m_{l}+p_{l}\right) q_{l}\right\} .
\end{aligned}
$$

The theta functions and its derivatives were approximated numerically via finite sums, $\left|m_{1,2}\right| \leq N$, in Matlab. For Riemann matrices in the fundamental domain, values of $N=$ 3,4 were sufficient to reach machine precision. ${ }^{2}$ We covered Gottschling's domain with a cartesian grid with 40 points in each direction (since we restricted the analysis to positive values of $x_{3}$ only 20 points for this direction were needed in the computation to obtain the same resolution as for the other $x$-directions). It turns out that roughly $40 \%$ of the points lie inside the fundamental domain. The modular invariants were only calculated at these points. In a first step we numerically identify values close to the minimum of the gradient for a given value of $y_{1}$ (within 0.01 of the respective minimum, in total more than 8,000 terms). The found values are used as an initial guess to search for a zero of the gradient. To identify the stationary points we use the algorithm of [21] which is implemented as the function fminsearch in Matlab.

It turns out that four out of six critical points found numerically coincide to the order of machine precision with the points listed in Theorem 6 (which provides an additional test of the numerics). Two other points are $\operatorname{Sp}(4, \mathbf{Z})$ equivalent and correspond to some Riemann surface from the $D_{3}$ family. All these points are located on the boundary of the fundamental domain.

The full list of the critical points found in one half of Gottschling's domain, where $x_{3} \geq 0$, reads:

1. A point which can be identified with

$$
\left(\begin{array}{cc}
\eta & (\eta-1) / 2 \\
(\eta-1) / 2 & \eta
\end{array}\right),
$$

where $\eta=(1+2 \sqrt{2} i) / 3$; this point is $\operatorname{Sp}(4, \mathbf{Z})$ equivalent to the point $\mathbf{B}_{1}$ of Theorem 6 , the Burnside curve. The value of $\mathcal{F}$ for this point equals 0.3106 (we only give here 4 digits for the sake of presentation though at least 13 digits are known); this is the global maximum of the function $\mathcal{F}$. This result is confirmed by the computation of the signature of the Hessian which equals $(0,6)$.

2 Matlab works with a precision of 16 digits; due to rounding errors machine precision is typically limited to 14 digits. 
2. A point which can be identified with the point $\mathbf{B}_{2}$ of Theorem 6, i.e., with the matrix of $b$-periods of the $D_{6}$ curve. The value of $\mathcal{F}$ at this point equals 0.2507 ; the signature of the Hessian is $(3,3)$.

3. Two points which can be identified with the matrices of $b$-periods of the $\mathbf{Z}_{5}$ curve:

$$
\mathbf{B}=\left(\begin{array}{cc}
\epsilon_{5} & \epsilon_{5}+\epsilon_{5}^{3} \\
\epsilon_{5}+\epsilon_{5}^{3} & -\epsilon_{5}^{4}
\end{array}\right), \quad\left(\begin{array}{cc}
-\epsilon_{5}^{4} & \epsilon_{5}+\epsilon_{5}^{3} \\
\epsilon_{5}+\epsilon_{5}^{3} & \epsilon_{5}
\end{array}\right),
$$

where $\epsilon_{5}=\exp (2 \pi i / 5)$. These two points are $\operatorname{Sp}(4, \mathbf{Z})$ equivalent to the point $\mathbf{B}_{3}$ of Theorem 6. The value of the function $\mathcal{F}$ at these points equals 0.2912 .

The modular equivalent points (4.14) belong to the boundary of the fundamental domain, and coincide when the boundary points get appropriately identified. The signature of the Hessian at these points equals $(2,4)$.

4. Two $\operatorname{Sp}(4, \mathbf{Z})$ equivalent points

$$
\mathbf{B}=\left(\begin{array}{cc}
1.0517 i & \pm 0.5+0.5259 i \\
\pm 0.5+0.5259 i & 1.0517 i
\end{array}\right)
$$

(we give the components of this matrix with higher precision below) which belong to the $D_{3}$ family. The value of $\mathcal{F}$ at these points equals 0.3011 . These points also belong to the boundary of $\mathcal{G}$. The signature of the Hessian equals $(1,5)$.

The symmetry of $\mathcal{F}$ implies the existence of further critical points for negative values of $x_{3}$ in the fundamental domain except for the points above with $x_{3}=0$. However these additional points are again related to their originals by $\operatorname{Sp}(4, \mathbf{Z})$ transformations, since all of these points belong to the boundary of $\mathcal{G}$. Therefore this does not contradict the fact that $\mathcal{G}$ is a fundamental domain of $\operatorname{Sp}(4, \mathbf{Z})$.

Remark 1 The existence of the critical point (4.15) of $\mathcal{F}$ might appear surprising since it does not follow from Theorem 6: this is not a Riemann surface with a large automorphism group. This phenomenon is also new in comparison with genus one, where both critical points of $f$ (2.1) correspond to curves with large automorphism groups. However the existence of such a point in genus two follows from the mass formula for the virtual (orbifold) Euler characteristic of $\mathcal{M}$ (see [30,31] and Sect. 6 below). In [31], too, a point from the $D_{3}$ family was identified as a critical of the function syst. We do not know whether the critical $D_{3}$ curve from [31] coincides with (4.15). In our attempt to understand whether the point (4.15) has a universal character we considered another $\operatorname{Sp}(4, \mathbf{Z})$ invariant function on $\mathcal{H}$ - the absolute value of the first Igusa invariant [13]. Numerical analysis shows that the curve (4.15) is no longer critical for this new function on $\mathcal{M}$. Therefore it remains an interesting problem to find some geometrical interpretation of the curve (4.15) (for instance the analogous curve of [31], which is critical for syst, was claimed to be arithmetic). The value of $r$ in equation (3.31) defining the extremal $D_{3}$ curve can be computed by using Rosenhain's formulas (see, e.g., [6]) for branch points in terms of the matrix of $b$-periods; this value is given by (all digits given below are reliable, except for the last two):

$$
r=0.22373907612077
$$




\section{$5 \mathcal{F}$ on different strata of $\mathcal{M}$}

\subsection{Curves with $\mathbf{Z}_{2}$ symmetry}

For the curves with $\mathbf{Z}_{2}$ reduced symmetry group (3.6), when the matrix of $b$-periods for the canonical basis of cycles shown in Fig. 3, has the form of (3.12), it is possible to express the function $\mathcal{F}(1.1)$ in terms of elliptic theta functions of the moduli $\mathbf{x}$ and $\mathbf{y}$ by using the reduction formula for genus two theta functions. The result is given by the following lemma:

Lemma 6 Let the matrix of b-periods of a genus two curve have the form (3.12). Then the function $\mathcal{F}(1.1)$ can be represented in terms of genus one theta functions as follows:

$$
\mathcal{F}=\frac{1}{4} f^{3}(\mathbf{x}) f^{3}(\mathbf{y})(\Im \mathbf{x})(\Im \mathbf{y}) \mid\left(\vartheta_{3}^{4}(\mathbf{x}) \vartheta_{4}^{4}(\mathbf{y})-\vartheta_{4}^{4}(\mathbf{x}) \vartheta_{3}^{4}(\mathbf{y}) \mid\right.
$$

where $f(\mathbf{x})$ is given by $(2.1) ; \vartheta_{i}(\mathbf{x}), i=2,3,4$ are the genus one theta constants of module $\mathbf{x}$.

Proof When the matrix of $B$-periods has the form (3.12), the genus two theta function decomposes into a combination of elliptic theta functions with moduli $2 \mathbf{x}$ and $2 \mathbf{y}$ : (the first argument of the theta functions below is 0 )

$$
\Theta\left[\begin{array}{ll}
a & b \\
c & d
\end{array}\right](\mathbf{B})=\sum_{e \in\{0,1 / 2\}} \vartheta\left[\begin{array}{c}
(a+b) / 2+e \\
c+d
\end{array}\right] \vartheta(2 \mathbf{y})\left[\begin{array}{c}
(a-b) / 2+e \\
c-d
\end{array}\right](2 \mathbf{x})
$$

By regrouping the ten theta constants from (1.1) into five pairs, and by using the inverse binary addition formula for elliptic theta functions:

$$
\vartheta\left[\begin{array}{l}
a \\
b
\end{array}\right](2 \mathbf{x}) \vartheta\left[\begin{array}{l}
c \\
b
\end{array}\right](2 \mathbf{x})=\frac{1}{2} \sum_{d \in\{0,1 / 2\}} \exp (-i \pi a d) \vartheta\left[\begin{array}{l}
a+c \\
b+d
\end{array}\right](\mathbf{x}) \vartheta\left[\begin{array}{c}
a-c \\
d
\end{array}\right](\mathbf{x}),
$$

we get (5.1) after elementary manipulations.

It is clear that the Burnside curve is also the absolute maximum of $\mathcal{F}$ on $\mathcal{M}\left(\mathbf{Z}_{2}\right)$; the $D_{6}$ curve and the extremal $D_{3}$ curve must also be critical points of $\mathcal{F}$ on $\mathcal{M}\left(\mathbf{Z}_{2}\right)$ (the $\mathbf{Z}_{5}$ curve does not belong to $\mathcal{M}\left(\mathbf{Z}_{2}\right)$ ). The question whether $\mathcal{F}$ has critical points on $\mathcal{M}\left(\mathbf{Z}_{2}\right)$ in addition to these three, can also be given only a numerical (but rather conclusive) answer.

Taking into account Theorem 3 and Lemma 2, we study numerically the behavior of $\mathcal{F}$ and $\operatorname{grad} \mathcal{F}$ when $(\mathbf{x}, \mathbf{y}) \in \Omega \times \Omega(2)$. The numerical analysis with 100 points in each direction and a subsequent refined analysis as described above identifies the following critical points on $\Omega \times \Omega(2)$, all of which coincide up to $\operatorname{Sp}(4, \mathbf{Z})$ transformations with one of the four critical points of $\mathcal{F}$ on the whole $\mathcal{M}$ :

1. The point which coincides with the matrix of $b$-periods of the Burnside curve:

$$
\mathbf{x}=\sqrt{2} i \quad \mathbf{y}=\frac{2}{3}+\sqrt{2} i .
$$

This point, of course, gives the global maximum of $\mathcal{F}$ on $\mathcal{M}\left(\mathbf{Z}_{2}\right)$. The signature of the Hessian at this point is $(0,4)$.

2. Five points corresponding to the $D_{6}$ curve. The first point coincides with the point $\mathbf{B}_{2}$ of Theorem 6. The following four points which also belong to $\Omega \times \Omega(2)$ are $\operatorname{Sp}(4, \mathbf{Z})$ 
equivalent to $\mathbf{B}_{2}$ (i.e., they also represent the $D_{6}$ curve):

$$
\begin{array}{ll}
\mathbf{x}= \pm \frac{1}{2}+\frac{i \sqrt{3}}{2} & \mathbf{y}=\mp \frac{1}{2}+\frac{i \sqrt{3}}{2} \\
\mathbf{x}= \pm \frac{3}{2}+\frac{i \sqrt{3}}{2} & \mathbf{y}=\mp \frac{1}{2}+\frac{i \sqrt{3}}{2} \\
\mathbf{x}= \pm \frac{3}{2}+\frac{i \sqrt{3}}{2} & \mathbf{y}=\mp \frac{1}{2}+\frac{i \sqrt{3}}{2}
\end{array}
$$

The signature of the Hessian at these points is $(2,2)$.

3. Three points which are $\operatorname{Sp}(4, \mathbf{Z})$ equivalent to the critical $D_{3}$ curve (4.15) found on the full $\mathcal{M}$ :

$$
\begin{aligned}
& \left(\begin{array}{cc}
0.3835+0.7874 i & -0.4339+0.2114 i \\
-0.4339+0.2114 i & 0.3835+0.7874 i
\end{array}\right), \\
& \left(\begin{array}{cc}
1.0517 i & -0.5+0.5259 i \\
-0.5+0.5259 i & 1.0517 i
\end{array}\right) \\
& \left(\begin{array}{cc}
0.5+1.0517 i & 0.5259 i \\
0.5259 i & 0.5+1.0517 i
\end{array}\right)
\end{aligned}
$$

The signature of the Hessian at these points is $(1,3)$.

Remark 2 We see that the fundamental domain $\Omega \times \Omega(2)$ is indeed bigger than the moduli space $\mathcal{M}\left(\mathbf{Z}_{2}\right)$ : there are equivalences between different points of $\Omega \times \Omega(2)$ given by $\operatorname{Sp}(4, \mathbf{Z})$ transformations which do not correspond to any $\Gamma \times \Gamma$ (2) transformation on $\Omega \times \Omega(2)$.

\subsection{Curves with $D_{2}$ symmetry}

The matrices of $b$-periods (3.26) of the $D_{2}$ curves form a subfamily of the two-parametric family (3.12) with $\mathbf{y}=\mathbf{x}-1$; since $\vartheta_{3,4}(\mathbf{x}-1)=\vartheta_{4,3}(\mathbf{x})$, we have in this case

$$
\mathcal{F}=\frac{1}{4} f^{6}(\mathbf{x})(\Im \mathbf{x})^{2} \mid\left(\vartheta_{4}^{8}(\mathbf{x})-\vartheta_{3}^{8}(\mathbf{x}) \mid\right.
$$

due to Lemma 4, the moduli space $\mathcal{M}\left(D_{2}\right)$ in the variable $\mathbf{x}$ coincides with the fundamental domain $\Omega_{0}(2)+$ of the group $\Gamma_{0}(2)+$.

The plot of $\mathcal{F}$ in $\Omega_{0}(2)+$ is shown in Fig. 11 .

It is clear that the points corresponding to the Burnside and to the $D_{6}$ curves, which are critical points of $\mathcal{F}$ from a six-dimensional point of view, are also critical points of $\mathcal{F}$ on $\mathcal{M}\left(D_{2}\right)$ from a two-dimensional point of view. No other critical points of $\mathcal{F}$ in $\mathcal{M}\left(D_{2}\right)$ were found.

Namely for $\mathbf{x} \in \Omega_{0}(2)+$ we find the following critical points (see Fig. 7):

1. Two points corresponding to the Burnside curve are found at $\mathbf{x}=i / \sqrt{2}$ and $\mathbf{x}=i / \sqrt{2}+1$; the first of these values gives the point $\mathbf{B}_{1}$ of Theorem 6 . This is the absolute maximum of $\mathcal{F}$, and the signature of the Hessian is $(0,2)$.

2. The point corresponding to the $D_{6}$ curve is given by the value $\mathbf{x}=\frac{1}{2}+i \frac{\sqrt{3}}{2}$. The signature of the Hessian at this point is $(1,1)$.

The point of intersection of the two circles limiting the fundamental domain is $\mathbf{x}=$ $1 / 2+i / 2$; at this point the genus two Riemann surface splits into two tori; the function $\mathcal{F}$ vanishes at this point, as well as at $\mathbf{x} \rightarrow i \infty$. 


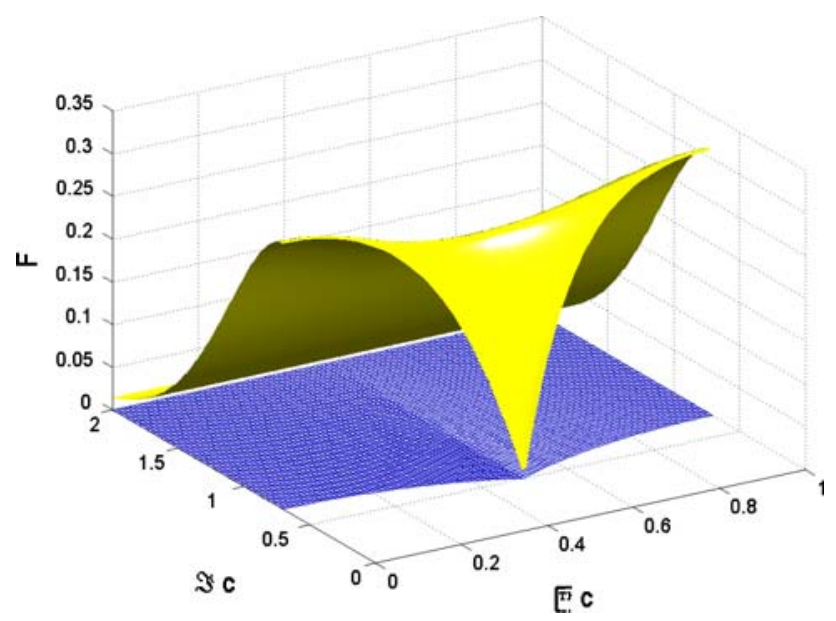

Fig. $11 \mathcal{F}(\sigma)$ in the fundamental domain $\Omega_{0}(2)+$

\subsection{Curves with $D_{3}$ symmetry}

The matrices of $b$-periods (3.35) of this family form a subfamily of the two-parametric family (3.12) with $\mathbf{x}=3 \sigma$ and $\mathbf{y}=\sigma$; then

$$
\mathcal{F}(\sigma)=\frac{3}{4} f^{3}(\sigma) f^{3}(3 \sigma)(\Im \sigma)^{2} \mid\left(\vartheta_{4}^{4}(\sigma) \vartheta_{3}^{4}(3 \sigma)-\vartheta_{3}^{4}(\sigma) \vartheta_{4}^{4}(3 \sigma) \mid ;\right.
$$

due to Lemma 5 the moduli space $\mathcal{M}\left(D_{3}\right)$ in the variable $\sigma$ coincides with the fundamental domain $\Omega_{0}(3)+$ of the group $\Gamma_{0}(3)+$.

The plot of $\mathcal{F}$ in $\Omega_{0}(3)+$ is shown in Fig. 12. It is clear that the points corresponding to the Burnside, the $D_{6}$ and the critical $D_{3}$ curves, which are critical points of $\mathcal{F}$ from a sixdimensional point of view, are also critical functions of $\mathcal{F}$ on $\mathcal{M}\left(D_{3}\right)$ from a two-dimensional point of view. No other critical points (i.e., such that the two-dimensional gradient vanishes) of $\mathcal{F}$ in $\mathcal{M}\left(D_{3}\right)$ were found numerically.

Namely we find for $\sigma \in \Omega_{0}$ (3) + the following critical points (see Fig. 9):

1. Two points corresponding to the Burnside curve are given by

$$
\sigma=\frac{1}{3}+\frac{i \sqrt{2}}{3}, \quad \sigma=\frac{2}{3}+\frac{i \sqrt{2}}{3} .
$$

the corresponding matrices of $b$-periods are related by $\operatorname{Sp}(4, \mathbf{Z})$ transformations to the point $\mathbf{B}_{1}$ of Theorem 6 . The signature of the Hessian is $(0,2)$.

2. Two points corresponding to the $D_{6}$ curve: $\sigma=i / \sqrt{3}$ and $\sigma=1+i / \sqrt{3}$; the first of these values gives the point $\mathbf{B}_{2}$ of Theorem 6 . The signature of the Hessian is $(1,1)$.

3. The point corresponding to the extremal $D_{3}$ curve is given by:

$$
\sigma=0.5+0.5259 i \text {. }
$$

The corresponding matrices of $b$-periods are $\operatorname{Sp}(4, \mathbf{Z})$ equivalent to the matrices of $b$-periods (4.15). The signature of the Hessian is $(1,1)$.

The point of intersection of the two circles limiting the fundamental domain $\sigma=\frac{1}{2}+\frac{i}{2 \sqrt{3}}$ is the boundary point of the moduli space, where the genus two surface splits into two tori; according to Lemma $3, \mathcal{F}$ vanishes there. 


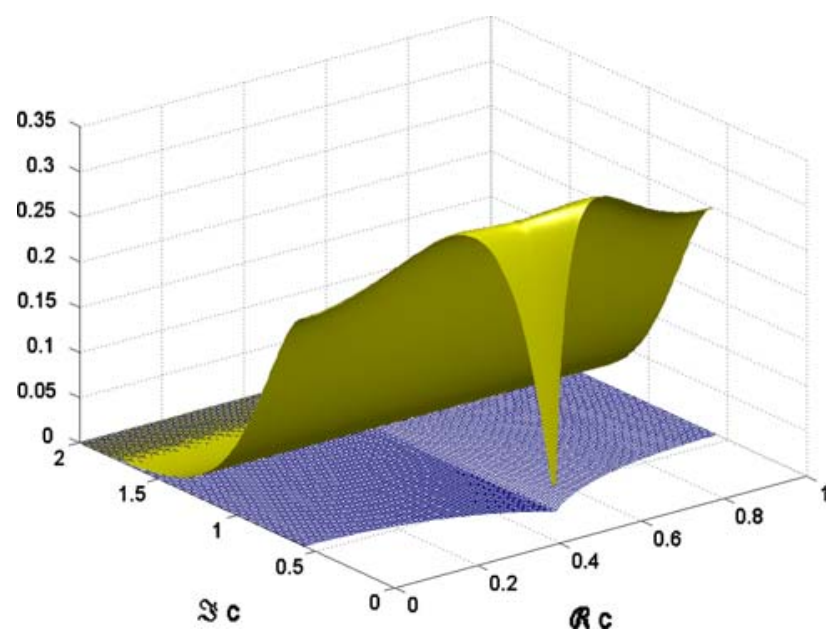

Fig. 12 Plot of $\mathcal{F}$ in the fundamental domain $\Omega_{0}(3)+$

\section{$6 \mathcal{F}$ and Euler characteristics of moduli spaces}

Given a function on the moduli space with non-degenerate critical points, it is natural to ask whether one can use this function as a Morse function, i.e., whether one can extract topological information (say, the Euler characteristic, which we shall discuss here) about the space. Since $\mathcal{M}$ (as well as its symmetric strata) is an orbifold, one can define many different Euler characteristics of $\mathcal{M}$ (see, for example, [5,12]). We shall speak here about the orbifold Euler characteristic in the sense of [34] defined as follows.

Let $X$ be a manifold (perhaps, with non-trivial boundary $\partial X$ ); let $\mathcal{G}$ be a group acting on $X$ effectively and properly discontinuously. Consider an arbitrary proper cell division of the orbifold $\mathcal{M}=X / G: X=\bigcup_{j=1}^{n} X_{j}$ (the cell division is called proper if all points of a given cell $X_{j}$ have the same stabilizer $G_{j}$ ). Then the orbifold Euler characteristic of $\mathcal{M}$ is defined as follows:

$$
\chi_{o}(\mathcal{M})=\sum_{j=1}^{n} \frac{(-1)^{\operatorname{dim} X_{j}}}{\#\left(G_{j}\right)}
$$

Suppose now $\mathcal{F}: \quad X \rightarrow \mathbf{R}$ to be a function on $X$ vanishing on the boundary $\partial X$, invariant under the action of the group $\mathcal{G}$ such that all critical points of $f$ are isolated and non-degenerate. The set of critical points of the function $\mathcal{F}$ must be invariant under the action of the group $\mathcal{G}$; let us assume that this set consists of a finite number $N$ of orbits; denote by $P_{1}, \ldots, P_{N}$ the critical points representing each orbit. Denote by $i_{1}, \ldots, i_{N}$ the indices of the critical points $P_{1}, \ldots, P_{N}$ (i.e., the numbers of negative eigenvalues of the corresponding Hessians). Denote by $\operatorname{Stab}\left(P_{j}\right)$ the stabilizer of the critical point $P_{j}$, i.e., the subgroup of $\mathcal{G}$ leaving the point $P_{j}$ invariant; the order of the subgroup is denoted by \#( $\left.\operatorname{Stab}\left(P_{j}\right)\right)$.

Then the orbifold Euler characteristic can be expressed via the following "mass formula" (see $[2,31]$ and references therein):

$$
\chi_{o}(X / \mathcal{G})=\sum_{j=1}^{N} \frac{(-1)^{i_{j}}}{\#\left(\operatorname{Stab}\left(P_{j}\right)\right)}
$$


We recall that if $f$ is a Morse function with a finite number of critical points on a manifold, then the ordinary Euler characteristic is equal to $\sum(-1)^{i_{j}}$, where $i_{j}$ are indices of the critical points.

A moduli space $\mathcal{M}_{g}$ can be represented as the factor $\mathcal{T}_{g} / \mathcal{G}_{g}$ of the Teichmüller space $\mathcal{T}_{g}$ by the mapping class group $\mathcal{G}_{g}$. Let $\mathcal{F}$ be a $C^{1}$ function on $\mathcal{T}_{g}$ invariant with respect to the mapping class group $\mathcal{G}_{g}$; let $\mathcal{L}_{1}, \ldots, \mathcal{L}_{N}$ be Riemann surfaces representing the orbits of the critical points of $\mathcal{F}$ on $\mathcal{T}_{g}$. Then for $g>2 \operatorname{Stab}\left(\mathcal{L}_{j}\right)$ is $\operatorname{simply}$ the group $\operatorname{Aut}\left(\mathcal{L}_{j}\right)$ of (holomorphic) automorphisms of $\mathcal{L}_{j}$ and the mass formula (6.2) looks as follows:

$$
\chi_{o}\left(\mathcal{M}_{g}\right)=\sum_{j=1}^{N} \frac{(-1)^{i_{j}}}{\#\left(\operatorname{Aut}\left(\mathcal{L}_{j}\right)\right)} \quad g>2 .
$$

For $g=1,2$ any curve from $\mathcal{M}_{g}$ possesses the hyperelliptic involution, and the right-hand side of (6.3) should be multiplied by 2 , i.e.,

$$
\chi_{o}\left(\mathcal{M}_{g}\right)=2 \sum_{j=1}^{N} \frac{(-1)^{i_{j}}}{\#\left(\operatorname{Aut}\left(\mathcal{L}_{j}\right)\right)} \quad g=1,2 .
$$

\subsection{Genus one}

The moduli space $\mathcal{M}_{1}$ can be obtained by standard gluing of the boundary of the fundamental domain $\Omega$ (Fig. 4); as a result we get the sphere with one puncture (the asymptotic cylinder corresponding to $\sigma \rightarrow \infty$ ) and two orbifold points corresponding to $\sigma=i$ (of index 2) and $\sigma=e^{\pi i / 3}$ (of index 3). The ordinary Euler characteristic of this space is 1 .

The orbifold Euler characteristic of $\mathcal{M}_{1}$ equals $-1 / 6$, as follows from considering an obvious cell division; this result can be easily reproduced using the "mass formula" (6.4). Namely, the function $f(\sigma)(2.1)$ has two critical points: $\sigma=i$ and $\sigma=e^{2 \pi i / 3}$; the order of the automorphism group of the torus with period $i$ is 4 , and the order of the automorphism group of the torus with period $e^{2 \pi i / 3}$ is 6 ; thus the "mass formula" $(6.4)$ gives $2(1 / 6-1 / 4)=-1 / 6$.

\subsection{Genus two}

\subsubsection{Full moduli space}

The ordinary Euler characteristic of the moduli space $\mathcal{M}$ in genus two as well as of its symmetric strata (Bolza subspaces) were discussed in a recent paper [9]. In particular $\chi(\mathcal{M})=1$.

The orbifold Euler characteristic of $\mathcal{M}$ equals $-1 / 120$ [11]. On the other hand, computing the right-hand side of (6.4) for our function $\mathcal{F}$, we get (taking into account the indices of $\mathcal{F}$ on the Burnside, the $D_{6}$, the $\mathbf{Z}_{5}$ and the critical $D_{3}$ curves are equal to 6, 3, 4 and 5 respectively, and the corresponding number of automorphisms which are 48, 24, 10 and 12 respectively):

$$
2\left(\frac{1}{48}-\frac{1}{24}+\frac{1}{10}-\frac{1}{12}\right)=-\frac{1}{120},
$$

in agreement with [11] (this computation coincides with the one done in [31] where a similar analysis was performed for the function $s y s t$ ).

Theorem 7 Let $\mathcal{F}$ be any modular invariant smooth function on $\mathcal{H}$ vanishing on the boundary of the moduli space $\mathcal{M}=(\mathcal{H} \backslash \mathcal{D}) / \mathrm{Sp}(4, \mathbf{Z})$ and such that all of its critical points are non-degenerate. Then in addition to critical points at the three Riemann surfaces with large 
automorphism groups, $\mathcal{F}$ must have at least one more critical point within the $\mathrm{D}_{3}$ family (3.31) or the $D_{2}$ family (3.21).

Proof is elementary: subtracting any combination of $1 / 48,1 / 24$ and $1 / 10$ from $-1 / 240$ with different signs, we get 8 different numbers. All of them have too large denominators to arise only from curves which do not belong to the of $D_{2}$ and $D_{3}$ families.

Theorem 7 does not exclude the existence of other critical points of the function $\mathcal{F}$ such that their total contribution to the mass formula (6.4) vanishes. However, our numerical analysis shows that $\mathcal{F}$ has indeed only four critical points on $\mathcal{M}$ : three standard ones, and one more from the $D_{3}$ family, and all of them are non-degenerate.

\subsubsection{Symmetric strata}

Although we shall speak here only about the genus two case, most of the construction makes sense for an arbitrary genus. Denote by $\Sigma^{G}$ the set of Riemann surfaces having the group $G$ as their group of automorphisms. The space $\Sigma^{G}$ contains also Riemann surfaces whose groups of automorphisms has $G$ as a subgroup. We shall denote by the same letter the element of the group and the biholomorphic automorphism of the Riemann surface corresponding to this element. All the homeomorphisms below are assumed to be orientation preserving.

Consider a basepoint $\mathcal{L}_{0} \in \Sigma^{G}$ and define a $G$-symmetric marking of a surface $\mathcal{L} \in \Sigma^{G}$ as a homeomorphism

$$
\phi: \mathcal{L}_{0} \rightarrow \mathcal{L}
$$

such that for any element $g_{1}$ of $G$ the map $\phi g_{1} \phi^{-1}$ is homotopic to some $g_{2} \in G$. Two $G$-symmetrically marked surfaces $\mathcal{L}_{1}$ and $\mathcal{L}_{2}$ from $\Sigma^{G}$ with $G$-symmetric markings $\phi_{1}$ : $\mathcal{L}_{0} \rightarrow \mathcal{L}_{1}$ and $\phi_{2}: \mathcal{L}_{0} \rightarrow \mathcal{L}_{2}$ are said to be equivalent if there exists a biholomorphic map $h: \mathcal{L}_{1} \rightarrow \mathcal{L}_{2}$ which is homotopic to the map $\phi_{2} \phi_{1}^{-1}$.

The set of equivalence classes of $G$-symmetrically marked surfaces from $\Sigma^{G}$ is called $G$-special Teichmüller space and denoted by $\mathcal{T}^{G}$. We are not aware of any general results about the structure of these spaces; for genus two their description is given in [29].

We call a homeomorphism $\Phi: \mathcal{L}_{0} \rightarrow \mathcal{L}_{0}$ G-symmetric if for any $g_{1} \in G$ there exists $g_{2} \in G$ such that the homeomorphisms $\Phi g_{1}$ and $g_{2} \Phi$ are homotopic. Denote the group $\mathcal{G}_{G}^{\prime}$ to be the group of homotopy classes of all $G$-symmetric homeomorphisms $\Phi: \mathcal{L}_{0} \rightarrow \mathcal{L}_{0}$.

The group $\mathcal{G}_{G}^{\prime}$ acts on $\mathcal{T}^{G}$ as follows. Let $\mathcal{L}$ be a $G$-symmetrically marked surface with $G$-symmetric marking $\phi: \mathcal{L}_{0} \rightarrow \mathcal{L}$ and [ $\left.\Phi\right]$ be an element of $\mathcal{G}_{G}^{\prime}$ defined by a $G$-symmetric homeomorphism $\Phi: \mathcal{L} \rightarrow \mathcal{L}$. Then $[\Phi](\mathcal{L})$ is the same surface $\mathcal{L}$ marked as

$$
\phi \circ \Phi^{-1}: \mathcal{L}_{0} \rightarrow \mathcal{L}
$$

obviously, this marking is also $G$-symmetric. Let $I$ be a subgroup of $\mathcal{G}_{G}^{\prime}$ whose elements preserve any $\mathcal{L} \in \mathcal{T}^{G}$.

The G-special mapping class group $\mathcal{G}_{G}$ is defined as the factor-group $\mathcal{G}_{G}=\mathcal{G}_{G}^{\prime} / I$ (this definition excludes the hyperelliptic involution in genus two). The action of $\mathcal{G}_{G}^{\prime}$ on $\mathcal{T}^{G}$ gives rise to the properly discontinuous effective action of $\mathcal{G}_{G}$ on $\mathcal{T}^{G}$; the orbifold $\mathcal{M}^{G}=\mathcal{T}^{G} / \mathcal{G}_{G}$ is called the G-special moduli space.

Consider now the moduli spaces $\mathcal{M}\left(\mathbf{Z}_{2}\right)\left(=\mathcal{M}^{\mathbf{Z}_{2} \times \mathbf{Z}_{2}}\right), \mathcal{M}\left(D_{2}\right)\left(=\mathcal{M}^{D_{2} \times \mathbf{Z}_{2}}\right)$ and $\mathcal{M}\left(D_{3}\right)$ $\left(=\mathcal{M}^{D_{3} \times \mathbf{Z}_{2}}\right)$. These moduli spaces are factors of the corresponding special Torelli spaces $\mathcal{S}\left(\mathbf{Z}_{2}\right)$ (3.18), $\mathcal{S}\left(D_{2}\right)$ (3.29) and $\mathcal{S}\left(D_{3}\right)$ (3.38) by the special Torelli groups (3.19), $\Gamma_{0}(2)+$ and $\Gamma_{0}(3)+$, respectively. 
The universal covering of $\mathcal{S}\left(\mathbf{Z}_{2}\right)$ is the special Teichmüller space $\mathcal{T}\left(\mathbf{Z}_{2}\right)=H \times H$ [29]. The special Teichmüller spaces $\mathcal{T}\left(D_{2}\right)$ and $\mathcal{T}\left(D_{3}\right)$ are the universal coverings of $\mathcal{S}\left(D_{2}\right)$ and $\mathcal{S}\left(D_{3}\right)$, respectively; they both coincide with the upper half-plane $H$.

We denote corresponding special mapping class groups by $\mathcal{G}\left(\mathbf{Z}_{2}\right), \mathcal{G}\left(D_{2}\right)$ and $\mathcal{G}\left(D_{3}\right)$, respectively. Then the moduli spaces $\mathcal{M}\left(\mathbf{Z}_{2}\right), \mathcal{M}\left(D_{2}\right)$ and $\mathcal{M}\left(D_{3}\right)$ are represented as factors $\mathcal{T}\left(\mathbf{Z}_{2}\right) / \mathcal{G}\left(\mathbf{Z}_{2}\right), \mathcal{T}\left(D_{2}\right) / \mathcal{G}\left(D_{2}\right)$ and $\mathcal{T}\left(D_{3}\right) / \mathcal{G}\left(D_{3}\right)$, respectively.

Let $\mathcal{L}$ be a surface from $\mathcal{M}^{G}$ and let $F$ be its full group of holomorphic automorphisms. Then $G$ is a subgroup of $F$ and we denote by $N(G ; F)$ the normalizer of $G$ in $F$. Choose some $G$-symmetric marking $\phi: \mathcal{L}_{0} \rightarrow \mathcal{L}$ of $\mathcal{L}$ and denote by $\operatorname{Stab}(\mathcal{L})$ the stabilizer of the point $\mathcal{L} \in \mathcal{M}^{G}$ in the special mapping class group $\mathcal{G}_{G}$.

Lemma 7 The map $\kappa: N(G ; F) \rightarrow \operatorname{Stab}(\mathcal{L})$ defined by

$$
\kappa(f)=\left[\phi^{-1} f \phi\right],
$$

where $f \in N(G ; F)$ and $[\ldots]$ denotes the homotopy class, is the homomorphism of two groups.

Proof Let $\Phi:=\phi^{-1} f \phi$. To verify that $\kappa$ is the group homomorphism one has to show that

1. the homeomorphism $\Phi$ is $G$-symmetric

2. $[\Phi](\mathcal{L})=\mathcal{L}$.

Choose $g_{1} \in G$; then $\phi g_{1} \phi^{-1}=g_{0}$ for some $g_{0} \in G$. Since $f \in N(G ; F)$, there exists some $g_{2} \in G$ such that $f g_{0}=g_{2} f$. Therefore, $g_{2} f=f g_{0} \sim f \phi g_{1} \phi^{-1}$ (where $\sim$ denotes the homotopy equivalence) and there exists a $g_{3}$ such that

$$
\Phi g_{1} \sim \phi^{-1} g_{2} f \phi=\phi^{-1} g_{2} \phi \phi^{-1} f \phi \sim g_{3} \Phi,
$$

which proves the $G$-symmetry of $\Phi$.

To prove the second statement we write $\left(\phi \Phi^{-1}\right) \phi^{-1}=\phi \phi^{-1} f^{-1} \phi \phi^{-1}=f^{-1}$; thus the $G$-symmetric markings $\phi: \mathcal{L}_{0} \rightarrow \mathcal{L}$ and $\phi \circ \Phi^{-1}: \mathcal{L}_{0} \rightarrow \mathcal{L}$ are equivalent.

Lemma 8 The groups $N(G ; F) / G$ and $\operatorname{Stab}(\mathcal{L})$ are isomorphic.

Proof In view of the previous lemma it is sufficient to show that

1. $\kappa$ is a surjection,

2. $\operatorname{ker} \kappa=G$.

(1) Let $r \in \operatorname{Stab}(\mathcal{L})$, then $r=[\Phi]$, where [ $\Phi]$ is a $G$-symmetric homeomorphism $\Phi$ : $\mathcal{L}_{0} \rightarrow \mathcal{L}_{0}$ such that

$$
\left(\phi \Phi^{-1}\right) \phi^{-1} \sim h
$$

with some $h \in F$. To prove the surjectivity of $\kappa$ we have to show that $h \in N(G ; F)$ and $\kappa(h)=r$. Let $g_{1} \in G$, then

$$
g_{1} h \sim g_{1} \phi \Phi^{-1} \phi^{-1} \sim \phi g_{2} \Phi^{-1} \phi^{-1} \sim \phi \Phi^{-1} g_{3} \phi^{-1} \sim \phi \Phi^{-1} \phi^{-1} g_{4} \sim h g_{4},
$$

with some $g_{2}, g_{3}, g_{4} \in G$. Since two homotopic holomorphic automorphisms must coincide (see, e.g., Lemma 6.5.5 from [6]), we have $g_{1} h=h g_{4}$, and, therefore, $h \in N(G ; F)$. From (6.7) we get

$$
r=[\Phi]=\left[\phi^{-1} h \phi\right]=\kappa(h)
$$

as was stated. 
(2a) $G \subset \operatorname{ker} \kappa$.

We shall prove that for any $g \in G$ the element $\kappa(g)$ of the special mapping class group acts trivially on $\mathcal{T}^{G}$ and, therefore, is the unity (since the special mapping class group action is effective). Let $\mathcal{L}$ be a $G$-symmetrically marked surface with symmetric marking $\psi: \mathcal{L}_{0} \rightarrow \mathcal{L}$. Then

$$
\left(\psi \phi^{-1} g^{-1} \phi\right) \psi^{-1} \sim g^{-1}
$$

and, therefore, $\kappa(g)(\mathcal{L})=\mathcal{L}$.

(2b) $\operatorname{ker} \kappa \subset G$.

Let $f \in \operatorname{ker} \kappa$ and $\mathcal{L}$ be a generic surface admitting a holomorphic $G$-action (i.e. $\operatorname{Aut}(\mathcal{L})=$ $G$ ) and let $\phi: \mathcal{L}_{0} \rightarrow \mathcal{L}$ be a $G$-symmetric marking. Since $\kappa(f)=\mathbf{1}$, one has

$$
\left(\psi \phi^{-1} f^{-1} \phi\right) \psi^{-1} \sim g
$$

with some $g \in G$. Therefore $f^{-1} \sim \phi \psi^{-1} g \psi \phi^{-1} \sim g_{1}$ with some $g_{1} \in G$ and Lemma 6.5.5 from [6] implies that $f^{-1}=g_{1}$ and, therefore, $f \in G$.

Theorem 8 Let $\mathcal{F}$ be any smooth function on the special Teichmüller space $\mathcal{T}^{G}$ ( $G$ is any of the groups $D_{2} \times \mathbf{Z}_{2}, D_{3} \times \mathbf{Z}_{2}$ or $\mathbf{Z}_{2} \times \mathbf{Z}_{2}$ ) invariant with respect to the special mapping class group $\mathcal{G}_{G}$ and vanishing on the boundary of $\mathcal{T}^{G}$. Suppose that the function $\mathcal{F}$ has critical points at Riemann surfaces $\mathcal{L}_{k}$ which possess the automorphism groups $G_{k}$ respectively $(G$ is a subgroup of $\left.G_{k}\right)$. Denote by $H_{k}$ the factor-group of the normalizer $N\left(G, G_{k}\right)$ by $G$. Then the orbifold Euler characteristic of $\mathcal{M}^{G}$ is given by:

$$
\chi_{o}\left(\mathcal{M}^{G}\right)=\sum_{k} \frac{(-1)^{i_{k}}}{\#\left(H_{k}\right)}
$$

where $i_{k}$ is the index of the critical point $\mathcal{L}_{k}$ and $\#\left(H_{k}\right)$ is the order of the subgroup $H_{k}$.

Proof The mass formula (6.8) immediately follows from the mass formula (6.2) and Lemma 7.

Let us now consider the three symmetric strata of $\mathcal{M}_{2}$ separately.

Space $\mathcal{M}\left(D_{2}\right)$.

Let us compute $\chi_{o}\left(\mathcal{M}\left(D_{2}\right)\right)$ using the formula (6.8). Namely, let us enumerate the branch points $(0, \infty, 1, i,-1,-i)$ of the Burnside curve $z\left(z^{4}-1\right)$ by the numbers $(1,2,3,4,5,6)$ respectively. Then the automorphism $\mu_{1}: z \rightarrow i z$ acts on the set of branch points as the permutation $(3,4,5,6)$. The involution $\mu_{2}: z \rightarrow(z+1) /(z-1)$ acts as $(15)(23)(46)$; finally, the involution $z \rightarrow-1 / z$ acts as (12)(35).

The $D_{2}$ subgroup of $S_{4}$ is generated by the symmetries $z \rightarrow-z$ and $z \rightarrow r / z$ on the Riemann surface $z\left(z^{2}-1\right)\left(z^{2}-r^{2}\right)$. On the set of branch points $(0, \infty, 1, r,-1,-r)$ these symmetries act by the permutations (35)(46) and (12)(34)(56), respectively. The normalizer of the $D_{2}$ subgroup in $S_{4}$ contains one more generator: (12)(46). This element generates the factor-group $H$ of the normalizer by $D_{2}$ which is isomorphic to $\mathbf{Z}_{2}$. Thus $\#(H)$ for the Burnside curve equals 2.

Consider now the $D_{6}$ curve. Let us enumerate the branch points of the $D_{6}$ curve by $1, \ldots, 6$ starting from $z=1$ counterclockwise. Then the involution $\mu_{1}: z \rightarrow \epsilon_{6} z$ acts on this set as the permutation $(1,2,3,4,5,6)$; the involution $\mu_{2}: z \rightarrow-1 / z$ acts as the permutation (14)(23)(56). The $D_{2}$ subgroup of $D_{6}$ is generated by the involutions $z \rightarrow-z$ and $z \rightarrow-1 / z$ which correspond to the permutations (14)(25)(36) and (14)(23)(56). The 
normalizer of the $D_{2}$ subgroup in $D_{6}$ coincides with the subgroup itself, thus \# $(H)$ at $D_{6}$ curve equals 1 .

Thus, the formula (6.8) gives

$$
\chi_{o}\left(\mathcal{M}\left(D_{2}\right)\right)=-1+\frac{1}{2}=-\frac{1}{2} .
$$

Space $\mathcal{M}\left(D_{3}\right)$.

The $D_{3}$ symmetry on the curve $\left(z^{3}-1\right)\left(z^{3}-r^{3}\right)$ is generated by the transformations $z \rightarrow r / z$ and $z \rightarrow \epsilon_{3} z$. On the branch points of the Burnside curve these transformations act as the permutations $(2,5)(4,6)(1,3)$ and $(3,2,6)(1,5,4)$, respectively. The normalizer of the $D_{3}$ subgroup of $S_{4}$ coincides with the $D_{3}$ subgroup itself; thus at the $S_{4}$ curve \# $(H)=1$. On the other hand, $D_{3}$ is the subgroup of index 2 in $D_{6}$; thus it is a normal subgroup and at the $D_{6}$ curve $\#(H)=2$.

The formula (6.8) gives in this case

$$
\chi_{o}\left(\mathcal{M}\left(D_{3}\right)\right)=-1+1-\frac{1}{2}=-\frac{1}{2} .
$$

Space $\mathcal{M}\left(\mathbf{Z}_{2}\right)$.

To compute $\chi_{o}\left(\mathcal{M}\left(\mathbf{Z}_{2}\right)\right)$ via (6.8) we need to find the subgroups $H$ for the stationary points of $\mathcal{F}$, i.e., for the Burnside curve, the $D_{6}$ curve and the extremal $D_{3}$ curve.

The $\mathbf{Z}_{2}$ symmetry $z \rightarrow-z$ on the Burnside curve acts as the permutation (35)(46) of the branch points; using Maple we verify that the factor-group of the normalizer of this $\mathbf{Z}_{2}$ subgroup in $S_{4}$ by the $\mathbf{Z}_{2}$ subgroup is generated by the permutations (12)(34)(56) and (12)(46) and has order 4.

The $\mathbf{Z}_{2}$ symmetry on the $D_{6}$ curve is $z \rightarrow \epsilon_{6} / z$; on the branch points it acts as the permutation (12)(36)(45). The factor-group of the normalizer of the $\mathbf{Z}_{2}$ subgroup by the subgroup itself is another $\mathbf{Z}_{2}$ subgroup generated by the permutation (14)(25)(36); thus here $\#(H)=2$.

Consider a $D_{3}$ curve $\left(z^{3}-1\right)\left(z^{3}-r^{3}\right)$ and enumerate the branch points $\left(1, r, \epsilon_{3}, \epsilon_{3} r\right.$, $\left.\epsilon_{3}^{2}, \epsilon_{3}^{2} r\right)$ by the numbers $(1, \ldots, 6)$. Then the $D_{3}$ group is generated by the symmetries $z \rightarrow \epsilon_{3} z$ and $z \rightarrow r / z$; the corresponding permutations of the branch points are (135)(246) and (12)(36)(45); the permutation (12)(36)(45) defines the $\mathbf{Z}_{2}$ subgroup. The normalizer of this subgroup coincides with the subgroup itself; thus $\#(H)=1$.

Since the index of the Burnside curve in the $\mathbf{Z}_{2}$ family is 4 , the index of the $D_{6}$ curve is 2 and the index of the critical $D_{3}$ curve is 3 , the mass formula (6.8) gives:

$$
\chi_{o}\left(\mathcal{M}\left(\mathbf{Z}_{2}\right)\right)=\frac{1}{4}+\frac{1}{2}-1=-\frac{1}{4}
$$

\section{Summary and outline}

It is natural to ask whether the scheme presented here can be improved (in particular whether it can be made fully rigorous) or generalized to other functions on moduli spaces, or to other moduli spaces. All these questions are open; however one can make reasonable conjectures on the basis of this work. First it is natural to expect that any Riemann surface with large automorphism group is a critical point of any smooth function on the Teichmüller space, invariant with respect to the action of the mapping class group. As another confirmation of this conjecture one can prove this statement for the Klein curve, which reads in projective 
coordinates:

$$
x y^{3}+y z^{3}+z x^{3}=0 .
$$

According to [25], the matrix of $b$-periods of the Klein curve is given by:

$$
\mathbf{B}=\left(\begin{array}{ccc}
-\frac{1}{8}+\frac{3 \sqrt{7}}{8} i & -\frac{1}{4}-\frac{\sqrt{7}}{4} i & -\frac{3}{8}+\frac{\sqrt{7}}{8} i \\
-\frac{1}{4}-\frac{\sqrt{7}}{4} i & \frac{1}{2}+\frac{\sqrt{7}}{2} i & -\frac{1}{4}-\frac{\sqrt{7}}{4} i \\
-\frac{3}{8}+\frac{\sqrt{7}}{8} i & -\frac{1}{4}-\frac{\sqrt{7}}{4} i & \frac{7}{8}+\frac{\sqrt{3}}{8} i
\end{array}\right) ;
$$

the matrix

$$
\Sigma=\left(\begin{array}{cccccc}
1 & 1 & 1 & 1 & 0 & 0 \\
0 & -1 & -1 & -1 & 1 & 0 \\
0 & 1 & 0 & 1 & -1 & 0 \\
-1 & -1 & 0 & 0 & 0 & 0 \\
-1 & -1 & 0 & 0 & 0 & -1 \\
-1 & 0 & 0 & 0 & 0 & -1
\end{array}\right)
$$

belongs to the stabilizer of $\mathbf{B}$ in $\operatorname{Sp}(6, \mathbf{Z})$. Then in complete analogy to the proof of Theorem 6 we find that the spectrum of the six-dimensional analog of the matrix $\mathbb{A}$ (4.12) does not contain unity; thus any smooth function on the Teichmüller space invariant with respect to the mapping class group has a critical point at the Klein curve.

Another natural conjecture is that in genera 3 and higher there also exist analogs of the extremal genus two curve from the $D_{3}$ family which does not have a large group of automorphisms. The mass formula for the orbifold Euler characteristic can, perhaps, in some cases be helpful in proving the existence of such a curve similarly to the genus two case.

A further question is whether the determinant of the Laplacian in the Poincaré metric (which is maximal among all metrics within a given conformal class) has the same set of critical points and the same signatures of the Hessian as the determinant in the Bergman metric studied here. We suppose that the function $\operatorname{det} \Delta$ has only four critical points, similarly to $\operatorname{det} \Delta_{B}$, (the existence of three of them we have proved here) with the maximum at the Burnside curve, but the fourth critical point from the $D_{3}$ family is most probably different from the one for $\operatorname{det} \Delta_{B}$. We hope that either analytical or numerical analysis of these questions will be possible in the near future.

Finally we hope that the analysis of the global properties of appropriate analogs of $\operatorname{det} \Delta_{B}$ should be possible for other interesting spaces: Hurwitz spaces and the spaces of Abelian and quadratic differentials on Riemann surfaces. We hope that, say, for spaces of Abelian differentials $w$ on Riemann surfaces [17] the proper functional will be the determinant of the Laplacian operator in the flat metric with conical singularities given by $|w|^{2}$. Exact formulas for such determinants (and their analogs on Hurwitz spaces) obtained in [18,20] should enable at least an efficient numerical analysis of their global behavior; that could provide new geometrical information about these spaces having been much less studied than the moduli spaces of Riemann surfaces.

Acknowledgments We are grateful to L. Chekhov, D. Jakobson, K.-I. Yoshikawa and P. Zograf for interesting discussions. We thank C. Cummins for information about the fundamental domains of the groups $\Gamma_{0}(2,3)+$. The work of DK was partially supported by the Concordia Research Chair grant, NSERC and NATEQ. AK thanks the Max Planck institute for Mathematics in the Sciences in Leipzig for hospitality and excellent working conditions. DK thanks the Laboratory of Mathematical Physics of Université de Bourgogne. AK and DK thank the Max-Planck Institute for Mathematics in Bonn, where this work was completed, for warm hospitality. 


\section{References}

1. Apostol, T.: Modular Functions and Dirichlet Series in Number Theory, Graduate Texts in Mathematics, No. 41. Springer, Heidelberg (1976)

2. Ash, A.: On the existence of eutactic forms. Bull. Lond. Math. Soc. 12, 192-196 (1980)

3. Bochner, S., Martin, W.: Several Complex Variables. Princeton University Press, New Jersey (1948)

4. Bolza, O.: On binary sextics with linear transformations into themselves. Am. J. Math. 10, 47-60 (1888)

5. Bryan, J., Fulman, J.: Orbifold Euler characteristics and the number of commuting $m$-tuples in the symmetric group, math.CO/9712248

6. Buser, P., Silhol, R.: Geodesics, periods and equations of real hyperelliptic curves. Duke Math. J 108, 211-250 (2001)

7. Cummins, C.: Private communication

8. Ford, L.R.: Automorphic Functions. McGraw-Hill, New York (1929)

9. Getzler, E.: Euler characteristics of local systems on $\mathcal{M}_{2}$. Compos. Math. 132, 121-135 (2002)

10. Gottschling, E.: Explizite Bestimmung der Randflächen des Fundamentalbereiches der Modulgruppe zweiten Grades. Math. Ann. 138, 103-124 (1959)

11. Harer, J., Zagier, D.: The Euler characteristic of the moduli space of curves. Invent. Math. 85, 457-485 (1986)

12. Hirzebruch, F., Höfer, T.: On the Euler number of an orbifold. Math. Ann. 286, 255-260 (1990)

13. Igusa, J.: Arithmetic variety of moduli for genus two. Ann. Math. 72(2), 612-649 (1960)

14. Jakobson, D., Levitin, M., Nadirashvili, N., Nigam, N., Polterovich, I.: How large can the first eigenvalue be on a surface of genus two? math.SP/0509398. Intern. Math. Res. Notices, to appear

15. Jorgenson, J., Kramer, J.: Non-completeness of the Arakelov-induced metric on moduli space of curves. Manusc. Math. 119, 453-463 (2006)

16. Knizhnik, V.: Multiloop amplitudes in the theory of quantum strings and complex geometry. Soviet Phys. Uspekhi 32, 945-971 (1989)

17. Kontsevich, M., Zorich, A.: Connected components of the moduli spaces of holomorphic differentials with prescribed singularities. Invent. Math. 153, 631-678 (2003)

18. Kokotov, A., Korotkin, D.: Isomonodromic tau-function of Hurwitz Frobenius manifolds and its applications. Int. Math. Res. Not. 2006, Art. ID 18746, pp 1-34 (2006)

19. Kokotov, A., Korotkin, D.: Normalized Ricci flow and determinant of Laplacian. Lett. Math. Phys. 71, 241-242 (2005)

20. Kokotov, A., Korotkin, D.: Tau-functions on spaces of Abelian differentials and higher genus generalizations of Ray-Singer formula, preprint of MPI Leipzig, 46/2004

21. Lagarias, J.C., Reeds, J.A., Wright, M.H., Wright, P.E.: Convergence properties of the Nelder-Mead simplex method in low dimensions. SIAM J. Optim. 9(1), 112-147 (1998)

22. Lewittes, J.: Differentials and Metrics on Riemann surfaces. Trans. Am. Math. Soc. 139, 311-318 (1969)

23. Osgood, B., Phillips, R., Sarnak, P.: Extremals of determinants of Laplacians. J. Funct. Anal. 80, 148211 (1988)

24. Pollicott, M., Rocha, A.: A remarkable formula for the determinant of the Laplacian. Invent. Math. 130, 399-414 (1997)

25. Rauch, E., Lewittes, J.: The Riemann surface of Klein with 168 automorphisms, in Problems in analysis (papers dedicated to S.Bochner), pp 297-308. Princeton University Press, New Jersey (1970)

26. Ray, D.B., Singer, I.M.: Analytic torsion for complex manifolds. Ann. Math. 98(2), 154-177 (1973)

27. Quine, J.R., Sarnak, P. (eds.): Extremal Riemann surfaces, Contemporary Mathematics, 201. AMS (1997)

28. Sarnak, P.: Extremal geometries, in [27]

29. Schiller, J.: Moduli for special Riemann surfaces of genus two. Trans. Am. Math. Soc. 144, 95-113 (1969)

30. Schmutz Schaller, P.: Geometry of Riemann surfaces based on closed geodesics. Bull. AMS 35(3), 193214 (1998)

31. Schmutz Schaller, P.: Systoles and topological Morse functions for Riemann surfaces. J. Differ. Geom. 52, 407-452 (1999)

32. Siegel, C.L.: Symplectic Geometry. Academic Press, New York (1964)

33. Silhol, R.: Period matrices and the Schottky problem, in Topics on Riemann surfaces and Fuchsian groups 155-163, London Math. Soc. Lecture Note Ser., 287. Cambridge University Press, Cambridge (2001)

34. Thurston, W.: Geometry and topology of 3-manifolds, Princeton lectures (1978)

35. Ji, L., Wentworth, R.: Spectral convergence on degenerating surfaces. Duke Math. J. 66(3), 469-501 (1992)

36. Yoshikawa, K.: Discriminants of theta divisors and Quillen metrics. J. Differ. Geom. 52, $73-115$ (1999)

37. Yoshikawa, K.: $K 3$ surfaces with involution, equivariant analytic torsion, and automorphic forms on the moduli space. Invent. Math. 156(1), 53-117 (2004) 